\title{
Model uncertainty and endogenous volatility
}

\author{
William A. Branch ${ }^{\mathrm{a}, *}$, George W. Evans ${ }^{\mathrm{b}}$ \\ a University of California, Irvine, USA \\ ${ }^{\mathrm{b}}$ University of Oregon, USA
}

Received 19 April 2006; revised 26 October 2006

Available online 26 December 2006

\begin{abstract}
This paper identifies two channels through which the economy can generate endogenous inflation and output volatility, an empirical regularity, by introducing model uncertainty into a Lucas-type monetary model. The equilibrium path of inflation depends on agents' expectations and a vector of exogenous random variables. Following Branch and Evans agents are assumed to underparameterize their forecasting models [Branch, W., Evans, G.W., 2006a. Intrinsic heterogeneity in expectation formation. Journal of Economic Theory 127, 264-295]. A Misspecification Equilibrium arises when beliefs are optimal, given the misspecification, and predictor proportions are based on relative forecast performance. We show that there may exist multiple Misspecification Equilibria, a subset of which is stable under least squares learning and dynamic predictor selection. The dual channels of least squares parameter updating and dynamic predictor selection combine to generate regime switching and endogenous volatility.
\end{abstract}

(c) 2006 Elsevier Inc. All rights reserved.

JEL classification: C53; C62; D83; D84; E40

Keywords: Lucas model; Model uncertainty; Adaptive learning; Rational expectations; Volatility

\section{Introduction}

Time-varying volatility in inflation and GDP growth is an empirical regularity of the US economy. This observation is often described in the applied literature as a regime shift during the 1980s which resulted in a simultaneous decline in inflation and output volatility. The 'Great Moderation', econometrically identified by Stock and Watson (2003) and McConnell and Quiros

\footnotetext{
* Corresponding author.

E-mail address: wbranch@uci.edu (W.A. Branch).
} 
(2000), among others, is often associated with a change in the stance of monetary policy (e.g. Branch et al., 2006).

However, recent studies by Cogley and Sargent (2005a) and Sims and Zha (2006) present evidence that drifting and regime switching inflation and output volatility is a characteristic of the post-war period. Since the Great Moderation consists of a one-time simultaneous decline in volatility, and its timing coexists with changes in Federal Reserve policy, it seems natural to seek policy explanations of this particular event. Persistently evolving inflation volatility may not always go hand in hand with changes in Federal Reserve policy. In this paper, we demonstrate that drift and regime switching in volatility may arise endogenously through model uncertainty.

Private sector expectations of future economic variables play a key role in most monetary models (e.g. Woodford, 2003). In these self-referential models, agents' beliefs feed back positively onto the underlying stochastic process. Yet, there is no consensus among economists on how agents actually form their expectations. While Rational Expectations provides a natural benchmark, there is a rapidly expanding literature, e.g. Marcet and Sargent (1989), Brock and Hommes (1997), Sargent (1999), Evans and Honkapohja (2001), and Marcet and Nicolini (2003), which replaces rational expectations with statistical learning rules. This alternative approach, it is argued, is a reasonable description of agents' actual forecasting acumen because it assumes behavior consistent with econometric practice.

Branch and Evans (2006a), though, note that with computational costs and degree of freedom limitations, econometricians often underparameterize their forecasting models. It has long been recognized that Vector Autoregressive (VAR) models have degrees of freedom limitations. Recent work by Chari et al. (2005) argue that these limitations present obstacles to VAR researchers who try to uncover a model's stochastic structure from observable time-series variables. The approach in this, and our earlier, paper is to model agents as VAR econometricians who behave optimally given the restrictions imposed on them by the data. The previous paper, developed in the context of the cobweb model, derived heterogeneity as an equilibrium outcome when agents choose the dimension in which to underparameterize. The current paper revisits that approach, instead framing the analysis in a standard Lucas-type monetary model.

We confront agents with a list of underparameterized predictor functions. The economic model is self-referential in the sense that agents' expectations, a function of their underparameterization choice, depends on the underlying stochastic process which, in turn, depends on these beliefs. A Misspecification Equilibrium (ME) is a fixed point of this self-reinforcing process. Model uncertainty arises in the sense that agents pick the best-performing statistical model. In our framework, what constitutes the best performing model depends not only on the regressors of the model, but also on the forecasting model choices of other agents. Of course, there are other ways to treat model uncertainty, for instance uncertainty about parameters (Hansen and Sargent, 2005) or about the form of the monetary policy rule. However, we believe that econometric model uncertainty is an important component of agent behavior, and this paper studies its implications.

There are two primary results in the current paper: first, when there are multiple underparameterized models from which agents must choose one, there may exist multiple stable equilibria, each with distinct stochastic properties; second, when agents must adaptively learn the forecast accuracy of these models, the economy will generate endogenous variation in inflation and output volatility.

This paper specifies a simple monetary model in which the reduced-form representations of aggregate supply and aggregate demand depend on a vector of autoregressive exogenous disturbances, representing preference and technology shocks, and supply additionally depends on 
unanticipated price level changes. Motivated by the idea that cognitive and computing time constraints and degrees of freedom limitations lead agents to adopt parsimonious models, we impose that agents only incorporate a subset of these variables into their forecasting model. Following Branch and Evans (2006a), we require that these expectations are optimal linear projections given the underparameterization restriction and that agents only choose best performing statistical models. Despite the bounded rationality assumption, this remains in the spirit of Muth (1961) in the sense that for each statistical model the parameters are chosen optimally. An equilibrium in beliefs and the stochastic process is a Misspecification Equilibrium. An ME extends the notion of a Restricted Perceptions Equilibrium, which arise in the models of Evans, Honkapohja, and Sargent (1989), Evans and Honkapohja (2001), and Sargent (1999), to settings in which agents must choose their models. We show that in the Lucas model there exist multiple ME and, moreover, the ME with homogeneous expectations are stable under least squares learning.

One implication of our theoretical model is that in a real-time dynamic version of the model agents must simultaneously estimate the parameters of their forecasting model and choose the best model based on past experience. We show that when agents use least squares to estimate the parameters of their statistical model, and base forecast performance on average mean-square forecast error of the competing models, different Misspecification Equilibria, in each of which agents coordinate on one forecasting model, can be stable.

Most interestingly, "constant gain" dynamics lead to new and distinct results. Constant gain least squares algorithms place a greater (time-invariant) weight on more recent observations. Constant gain (or "perpetual") learning, has been studied, for example, by Sargent (1999), Cho et al. (2002) and Orphanides and Williams (2005a), who argue for the plausibility of this form of learning dynamics as a way in which agents would allow for possible structural change. In this paper we extend this idea in an important way: learning jointly about model parameters and model fitness. Model uncertainty arises via constant gain learning and dynamic predictor selection.

Extending constant gain learning to incorporate dynamic predictor selection, we identify two channels through which inflation and output volatility may evolve over time. The first channel is from the parameter drift induced by constant gain updating of the forecasting model parameters. Under constant gain learning, the parameters vary around their mean values, even if the economy remains at a single equilibrium. In addition, regime switching in inflation and output volatility can arise when the economy switches endogenously between high and low volatility equilibria. Thus, the second channel is through dynamic predictor selection when agents react more strongly to recent forecast errors than distant ones when assessing the fitness of a forecasting model. Through numerical simulations, we show that, when there is dynamic predictor selection and parameter drift, the dynamic paths of inflation and output are consistent with the empirical regularities identified by Cogley and Sargent (2005a) and Sims and Zha (2006). These numerical results should be thought of as a conceptual exercise rather than a realistic calibration. We argue, however, that the channels identified in this paper are novel and that they warrant further study to assess their relative contributions to explaining stochastic volatility.

Our paper builds upon Brock and Hommes (1997, 1998), who study dynamic predictor selection in deterministic models using a similar reduced form to the model used here. ${ }^{1}$ Branch and Evans (2006a) extend Brock and Hommes to a stochastic environment in which, in equilibrium,

\footnotetext{
1 Evans and Ramey (1992) examined dynamic predictor selection when agents choose between naive forecasts and more costly forecasts computed from a structural model.
} 
both the choice of forecasting model and the parameters of each predictor are determined simultaneously. In that paper, we show that an equilibrium can arise in which agents are distributed heterogeneously across forecasting models. The contribution of the current paper is to demonstrate the possibility of multiple equilibria in an economic model with positive expectational feedback, and to show that dual learning in parameters and predictor selection can therefore generate the type of dynamics arguably present in macroeconomic time series.

This paper proceeds as follows. Section 2 presents evidence of time-varying volatility in the US economy. Section 3 presents the Lucas model with model uncertainty. Sections 4 and 5 consider the model under real-time learning. Section 6 concludes.

\section{Inflation and output volatility in the US}

\subsection{An empirical overview}

In the applied literature there is widespread consensus that during the 1980s there was a decline in economic volatility. An array of econometric techniques to identify the regime shift have been employed by Bernanke and Mihov (1998), Kim and Nelson (1999), Kim et al. (2004), McConnell and Quiros (2000), Sensier and van Dijk (2004), and Stock and Watson (2003). Recently, though, Cogley and Sargent (2005a) and Sims and Zha (2006) have identified repeated regime shifting economic volatility in US inflation and GDP growth. While Cogley-Sargent and Sims-Zha are interested in characterizing changing monetary policy over the period they make a striking finding: during the post-war period there is persistent stochastic volatility in the economy.

Conventional macroeconomic models, however, are unable to generate persistent stochastic volatility without directly assuming either exogenous disturbances following a Markov chain or exogenous changes in policy. In this paper, we present a model capable of generating such volatility endogenously via an adaptive learning and dynamic predictor selection process in a setting where agents may choose between competing underparameterized forecasting models. First, though, this section presents a brief, informal accounting of the nature of stochastic volatility in the economy.

Stochastic volatility is rigorously documented by Cogley and Sargent (2005a) and Sims and Zha (2006). Our purpose here is motivation and overview; we refer the reader to these other papers for formal econometric analysis. We detrend the log of real GDP using the HodrickPrescott filter since in the Lucas model below output is expressed as a log deviation from its trend value. Figure 1 plots conditional variances of inflation and (detrended) GDP for the period 1955:1-2004:2.

Owyang (2001) presents evidence that inflation follows an ARCH process. Figure 1 plots the conditional variances from an ARCH specification for inflation and log GDP to demonstrate the robustness of the finding that there is persistent stochastic volatility in both inflation and log GDP. To compute the conditional variances in Fig. 1 we estimated a $\operatorname{GARCH}(1,1)$ for an $\operatorname{AR}(4)$ model of inflation and log GDP. This follows exactly Owyang (2001) except that we also estimate a GARCH model for the volatility of $\log$ GDP. Figure 1 then plots the conditional variances from the GARCH models. ${ }^{2}$

\footnotetext{
2 Drifting and regime switching volatility is also evident in moving averages of unconditional variances as well as the econometric approaches of Sims-Zha, Cogley-Sargent, Justiniano and Primiceri (2006), and Fernandez-Villaverde and Rubio-Ramirez (2006).
} 

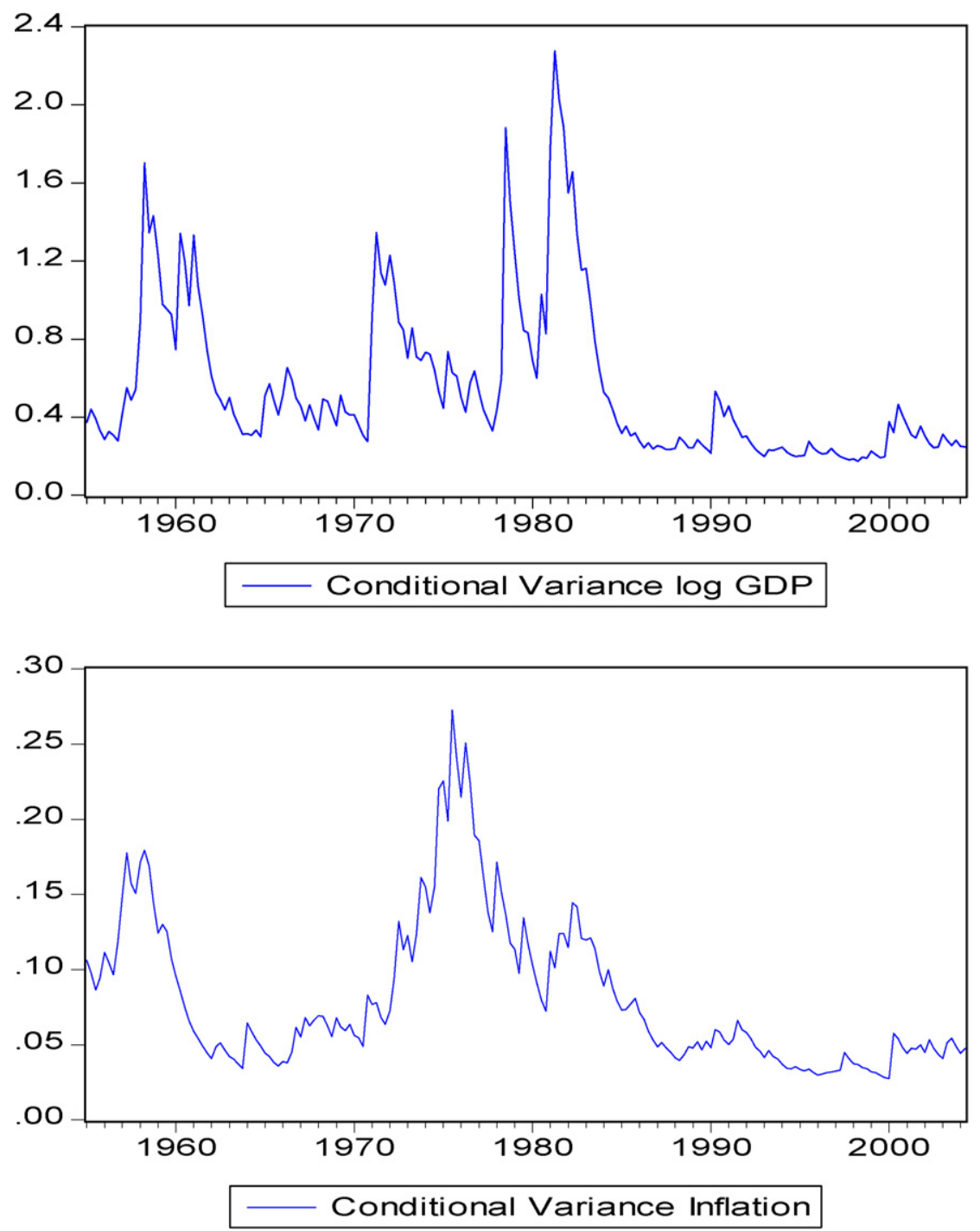

Fig. 1. Conditional variances from a GARCH(1, 1) model of an AR(4) for inflation and log GDP. Sample 1955:1-2004:2.

Inspection of Fig. 1 demonstrates the 'Great Moderation' emphasized by McConnell and Quiros (2000) and Stock and Watson (2003). About 1984 there was a simultaneous decline in the volatility of inflation and GDP. This empirical feature has generated considerable recent research into monetary policy's role in bringing about the observed economic stability. ${ }^{3}$ Figure 1 also demonstrates that the volatility of GDP and inflation varies over time. In particular, each series appears to move in tandem and alternate between high and low variance regimes. Sims and Zha (2006) find that 9 separate regimes fit the data best. This plot resembles the posterior mean

\footnotetext{
3 See, for example, Branch et al. (2006) and the references therein.
} 
estimates for the standard errors of the VAR innovations in Cogley and Sargent (2005a) over the period 1960-2000.

\subsection{Discussion}

Despite the attention given the Great Moderation, there seems to be little emphasis in the theoretical literature on accounting for the persistent stochastic volatility of the economy. Sims and Zha (2006) seek evidence in a change in the stance of monetary policy repeatedly across time. Sargent (1999) presents a theory of the rise and fall in inflation that is the result of drifting beliefs on the part of the government. ${ }^{4}$ Orphanides and Williams (2005a) account for the decline in volatility as a change in the stance of policy which pins down agents' drifting beliefs. ${ }^{5}$

In this paper, we develop another possible explanation that does not require policy changes. We present a model in which agents must choose between two alternative underparameterized models. We demonstrate the possibility of multiple coordinating equilibria with distinct stochastic properties across equilibria. We introduce model uncertainty by assuming that agents use constant gain least squares to estimate the parameters of their forecasting model. This introduces drifts into their beliefs as in Sargent (1999) and Orphanides and Williams (2005a). We also augment the model to allow agents to choose their forecasting model in real time based on a geometric weighted average of recent forecast performance. In this version of the model, agents switch persistently and endogenously between forecasting models. This induces the economy to switch between high and low inflation variance equilibria. Thus, we provide two possible sources of stochastic volatility: drifting beliefs and endogenous predictor selection.

It should be emphasized that we do not in any way discount the role for other explanations of persistent stochastic volatility, such as major shifts in macroeconomic policy. However, we do feel that the channels identified here, developed in the context of a very simple and otherwise well-behaved macroeconomic model, appear to be sufficiently powerful to merit serious empirical investigation in future research. The empirical results here motivate the conceptual exercise in Section 5. As such, we do not attempt to exactly replicate the conditional variances of Fig. 1.

\section{Model}

This section extends the Branch and Evans (2006a) cobweb model with misspecification to a Lucas-type monetary model. In Branch and Evans (2006a) firms choose planned output based on a misspecified forecasting model of the market price. Misspecification is modeled by confronting agents with a list of underparameterized models. Agents do, however, forecast optimally in the sense that they only choose the best performing statistical model. That paper establishes that, under appropriate joint conditions on the self-referential feature of the model and the exogenous disturbances, agents will necessarily be distributed heterogeneously across misspecified models.

Here we establish the existence of misspecification equilibria in a closely related Lucas-type monetary model. Later sections will address the dynamics of learning and predictor selection. Although the reduced form of the Lucas model is similar to the cobweb model, the slopes of the

\footnotetext{
4 Over a long stretch of time this would be expected to lead to periodic regime changes due to the 'escape' dynamics. For further discussion see Cho et al. (2002).

5 Recently, Justiniano and Primiceri (2006) and Fernandez-Villaverde and Rubio-Ramirez (2006) estimate business cycle models extended to incorporate stochastic volatility. Nevertheless, a theoretical channel to motivate the volatility remains an open issue.
} 
two models have opposite signs. The negative feedback of the cobweb model plays a central role in the existence of Intrinsic Heterogeneity. In the Lucas model the feedback from expectations is positive. The reinforcing aspect of expectations induces coordination by agents and raises the possibility of multiple equilibria. A striking feature of our results is that underparameterization makes multiple equilibria possible in a well-behaved model that has a unique equilibrium under fully rational expectations.

\subsection{Set-up}

The economy is represented by equations for aggregate supply (AS) and aggregate demand $(\mathrm{AD})$ :

$$
\begin{aligned}
& A S: q_{t}=\phi\left(p_{t}-p_{t}^{e}\right)+\beta_{1}^{\prime} z_{t}, \\
& A D: q_{t}=m_{t}-p_{t}+\beta_{2}^{\prime} z_{t}+w_{t}
\end{aligned}
$$

where $p_{t}$ is the log of the price level, $p_{t}^{e}$ is the $\log$ of expected price formed in $t-1, m_{t}$ is the log of the money supply, $q_{t}$ is the deviation of the log of real GDP from trend, $w_{t}$ is an i.i.d. zero-mean shock, and $z$ is a vector of potentially serially correlated disturbances with the properties described below. The AS curve yields a new classical Phillips curve, which has a venerable history in monetary economics, and has the same form as Lucas (1973), Kydland and Prescott (1977), Sargent (1999), and Woodford (2003). The AD equation is a simple quantity theory relationship.

The appendix provides one possible derivation of these reduced-form equations for AS and $\mathrm{AD}$ from a micro-founded model of heterogeneous expectations. We assume a yeoman farmer model along the lines of Woodford (2003): each farmer produces a differentiated good and sells it in a monopolistically competitive market. Assuming a money in the utility function framework yields a non-zero demand for money. Appendix A shows that in an economy with heterogeneous expectations across agents, the competitive equilibrium allocations have a representation in the reduced-forms above. The key assumption in this simple framework is that agents' money demand is interest inelastic yielding a quantity theory AD as shown in Walsh (2003). The purpose of this paper is to demonstrate the implications for the time-series properties of inflation and (detrended) GDP of learning and dynamic predictor selection. It would be interesting for future research to examine our approach in other AD frameworks.

Assume that the money supply follows, ${ }^{6}$

$$
\begin{aligned}
m_{t} & =p_{t-1}+\delta^{\prime} z_{t}+u_{t}, \\
z_{t} & =A z_{t-1}+\varepsilon_{t} .
\end{aligned}
$$

We assume for simplicity that $z_{t}$ is $(2 \times 1)$ and $\varepsilon_{t}$ is i.i.d. zero-mean with positive definite covariance matrix $\Sigma_{\varepsilon}$. The vector $z_{t}$ is also assumed to be a stationary process with the eigenvalues of $A$ inside the unit circle. The stochastic disturbance $z_{t}$ collects the serially correlated disturbances that affect aggregate supply, aggregate demand, and the money supply. The vectors $\beta_{1}, \beta_{2}, \delta$ determine which components of $z$ affect the respective reduced form relationships via (possible) zero components. The variable $u_{t}$ is a white noise money supply shock.

\footnotetext{
6 The form of the policy reaction function (implicitly) assumes the monetary authority does not observe $p_{t}, y_{t}$. This simple policy rule was adopted by Sargent (1987) and Evans and Ramey $(1992,2006)$. When $p_{t}$ is observable at $t$, a more general money supply rule is $m_{t}-p_{t}=-(1+\xi) \pi_{t}+\delta^{\prime} z_{t}+u_{t}$ with $\xi \geqslant 0$. This leads to the same reduced form as (1), with $\theta=\phi /(1+\phi+\xi)$.
} 
Denoting $\pi_{t}=p_{t}-p_{t-1}$ we can write the law of motion for the economy in its expectationsaugmented Phillips curve form

$$
\pi_{t}=\frac{\phi}{1+\phi} \pi_{t}^{e}+\frac{\left(\delta+\beta_{2}-\beta_{1}\right)^{\prime}}{1+\phi} z_{t}+\frac{1}{1+\phi}\left(w_{t}+u_{t}\right)
$$

or

$$
\pi_{t}=\theta \pi_{t}^{e}+\gamma^{\prime} z_{t}+v_{t}
$$

where $\theta=\frac{\phi}{1+\phi}, \gamma^{\prime}=\frac{\left(\delta+\beta_{2}-\beta_{1}\right)^{\prime}}{1+\phi}, v_{t}=\frac{1}{1+\phi}\left(w_{t}+u_{t}\right)$. Note, in particular, that $0 \leqslant \theta<1$. The cobweb model also takes the reduced form (1), but with $\theta<0$. This case is considered in Branch and Evans (2006a).

A rational expectations equilibrium (REE) is a stationary sequence $\left\{\pi_{t}\right\}$ which is a solution to (1) given $\pi_{t}^{e}=E_{t-1} \pi_{t}$, where $E_{t}$ is the conditional expectations operator. It is well known that (1) has a unique REE and that it is of the form

$$
\pi_{t}=(1-\theta)^{-1} \gamma^{\prime} A z_{t-1}+\gamma^{\prime} \varepsilon_{t}+v_{t}
$$

Output, in an REE, is a stationary process and does not display the time-series properties evident in the previous section. If instead agents only took one component of $z$ into account when forecasting inflation then the reduced form weights on the components of $z_{t}$ will change. Such a deviation from the REE (2) is a key insight of our model.

\subsection{Model misspecification}

This paper departs from the rational expectations hypothesis (RE) and imposes that agents are boundedly rational. One popular alternative to RE is to model agents as econometricians (e.g. Evans and Honkapohja, 2001). According to this literature, agents have a correctly specified model the parameters of which are estimated using a reasonable estimator. In many instances, these beliefs converge to RE. In practice, however, econometricians often misspecify their models. Professional forecasters often restrict the number of variables and/or lags to conserve degrees of freedom. Indeed, Chari et al. (2005) argue that econometric misspecification is central to the debate over identified Impulse Response Functions. Following Evans and Honkapohja (2001), Evans and Ramey (2006), and Branch and Evans (2006a), we argue that if agents are expected to behave like econometricians then they can also be expected to misspecify their models. We impose misspecification by forcing agents to underparameterize in at least one dimension. We follow Evans and Honkapohja (2001, Chapter 13), however, and impose that these underparameterized beliefs are optimal linear projections given the misspecification.

In this section we begin by defining the restricted perceptions equilibrium, given the misspecified models available and the proportion of agents using each model. In Section 3.3 we allow the model to endogenously determine the proportions and define a Misspecification Equilibrium. Then, in Section 4 we study the real-time dynamics of the model, with optimal projections replaced by least squares estimates and model choices determined by dynamic predictor selection based on recent performance.

Beliefs are formed from models that take one of the following forms:

$$
\begin{aligned}
& \pi_{t}^{e}=b^{1} z_{1, t-1}, \\
& \pi_{t}^{e}=b^{2} z_{2, t-1} .
\end{aligned}
$$


Because $z_{t}$ is a bivariate VAR(1) it is clear that (3)-(4) represent all possible nontrivial underparameterized models. Informally, we view the true economic process as being driven by a high dimensional exogenous process. That agents underparameterize, or approximate their econometric models, is a reasonable description of actual forecasting behavior. The assumption that $z_{t}$ is bivariate VAR(1) is, of course, made for analytical convenience. One can show the existence of Misspecification Equilibria, more generally, if $z_{t}$ is $n \times 1$ and follows a $\operatorname{VAR}(p)$. We impose that the parameters $b^{1}, b^{2}$ are formed as optimal linear projections of $\pi_{t}$ on $z_{i, t}$ for $i=1,2$. That is, beliefs satisfy the orthogonality condition

$$
E z_{i, t-1}\left(\pi_{t}-b^{i} z_{i, t-1}\right)=0 .
$$

This condition ensures that, in an equilibrium, agents' beliefs are consistent with the actual process in the sense that their forecasting errors are undetectable within their perceived model. When this occurs we say the model is at a Restricted Perceptions Equilibrium (RPE). ${ }^{7}$

Equilibria based on model misspecification that satisfy an orthogonality condition like (5) appear frequently in the literature. Rational expectations equilibria with limited information were studied in Sargent (1991) and Marcet and Sargent (1995), and Sargent (1999) examined the closely related concept of self-confirming equilibria. Consistent expectations equilibria, in which agents have linear beliefs consistent with a non-linear model, were developed in Hommes and Sorger (1998) and extended to stochastic models by Hommes et al. (2002) and Branch and McGough (2005). In Adam (2005a), one of the two possible model equilibria is an RPE. Evans and Ramey (2006) study optimal adaptive expectations in a Lucas-type model when the exogenous shock follows a complicated, unknown process. All of these approaches share the idea, exploited here, that agents are likely to misspecify their econometric model, but (in equilibrium) will do so optimally, given their misspecification. ${ }^{8}$

Of course, in the example we develop in this paper, with two underparameterized models, the misspecification would be easily identified by an experienced econometrician. To obtain tractable analytical results we develop our ideas using the simplest possible case, with a single endogenous variable of interest and a pair of exogenous variables following a VAR(1) process. Our analysis should be understood as a simplification of a much more complex economy with many endogenous variables of interest, driven by multiple exogenous observables following high order processes. We submit that the central themes of this paper would emerge in a more realistic model in which positive feedback from expectations plays an important role.

Because agents may be distributed heterogeneously across predictors, actual market beliefs for the economy are a weighted average of the individual beliefs

$$
\pi_{t}^{e}=n b^{1} z_{1, t-1}+(1-n) b^{2} z_{2, t-1}
$$

where $n$ is the proportion of agents who use model $1 .{ }^{9}$ Inserting these beliefs into (1) leads to

$$
\pi_{t}=\theta\left(n b^{1} z_{1, t-1}+(1-n) b^{2} z_{2, t-1}\right)+\gamma^{\prime} A z_{t-1}+\gamma^{\prime} \varepsilon_{t}+v_{t} .
$$

Or, by combining similar terms,

$$
\pi_{t}=\xi_{1} z_{1, t-1}+\xi_{2} z_{2, t-1}+\eta_{t}
$$

\footnotetext{
7 Adam (2005b) presents experimental evidence for approximate RPE in a bivariate macro model of output and inflation.

8 See also Guse (2005), who looks at "mixed expectations equilibria," in which a given proportion of agents may underparameterize the solution.

9 We identify model 1 as the model with the $z_{1, t}$ component and model 2 is defined symmetrically.
} 
where

$$
\begin{aligned}
& \xi_{1}=\gamma_{1} a_{11}+\gamma_{2} a_{21}+\theta n b^{1}, \\
& \xi_{2}=\gamma_{1} a_{12}+\gamma_{2} a_{22}+\theta(1-n) b^{2},
\end{aligned}
$$

$\eta_{t}=\gamma^{\prime} \varepsilon_{t}+v_{t}$, and $a_{i j}$ is the $i j$ th element of $A$. It follows from (5) and (6) that the optimal belief parameters are

$$
\begin{aligned}
& b^{1}=\xi_{1}+\xi_{2} \rho, \\
& b^{2}=\xi_{2}+\xi_{1} \tilde{\rho}
\end{aligned}
$$

where $\rho=E z_{1} z_{2} / E z_{1}^{2}$ and $\tilde{\rho}=E z_{1} z_{2} / E z_{2}^{2} \cdot{ }^{10}$ Note that the $\xi$ parameters are functions of $b$. These expressions for $b^{1}, b^{2}$ are versions of the standard 'omitted variable bias' formula. An RPE is a stationary process for $\pi_{t}$ which satisfies (6) with parameters $\xi_{1}, \xi_{2}$ that solve

$$
\left[\begin{array}{cc}
1-\theta n & -\theta n \rho \\
-\theta(1-n) \tilde{\rho} & 1-\theta(1-n)
\end{array}\right]\left[\begin{array}{l}
\xi_{1} \\
\xi_{2}
\end{array}\right]=A^{\prime} \gamma .
$$

A unique RPE exists if and only if the matrix which premultiplies the parameter vector is invertible. We formalize this invertibility condition below:

Condition $\Delta . \Delta \neq 0$ for all $n \in[0,1]$, where

$$
\Delta=1-\theta+\theta^{2} n(1-n)(1-\rho \tilde{\rho}) \text {. }
$$

Remark 1. Using the argument of Branch and Evans (2006a) it can be shown that Condition $\Delta$ is satisfied for all $0 \leqslant \theta<1$.

\subsection{Misspecification equilibrium}

A Misspecification Equilibrium (ME) is an RPE which jointly determines the fraction of agents using a given model. Below we formally define the equilibrium and present results on existence of ME.

We follow Brock and Hommes (1997) in assuming the map from predictor benefits to predictor choice is a multinomial logit (MNL) map. ${ }^{11}$ Brock and Hommes assume that agents base their predictor decisions on recent realizations of a deterministic process. In an ME we instead assume agents base their decisions on the unconditional moments of the stochastic process. Later when we introduce learning and dynamic predictor selection the predictor choice is based on an average of past realizations.

As in Evans and Ramey (1992), we assume agents seek to minimize their forecast MSE, i.e. we assume agents maximize

$$
E u=-E\left(\pi_{t}-\pi_{t}^{e}\right)^{2} .
$$

This assumption is reasonable in light of the linear RE literature and well-known results in leastsquares prediction theory. If $\pi_{t}^{e}$ is conditional on full information then RE would minimize the

\footnotetext{
10 The existence of these unconditional moments are guaranteed by the stationarity of $z_{t}$.

11 The use of the multinomial logit in discrete decision making is discussed extensively in Manski and McFadden (1981).
} 
expected mean-square error of one step-ahead forecasts. Thus, we preserve this structure when agents form optimal linear projections on a limited information set. The MNL approach leads to the following mapping, for each predictor $i=1,2$,

$$
n_{i}=\frac{\exp \left\{\alpha E u_{i}\right\}}{\sum_{j=1}^{2} \exp \left\{\alpha E u_{j}\right\}}
$$

Noting that $\sum_{j=1}^{2} n_{j}=1$, (8) can be rewritten

$$
n=\frac{1}{2}\left(\tanh \left[\frac{\alpha}{2}\left(E u_{1}-E u_{2}\right)\right]+1\right) \equiv H_{\alpha}\left(E u_{1}-E u_{2}\right)
$$

where $H_{\alpha}: \mathbb{R} \rightarrow[0,1]$.

The parameter $\alpha$, called the "intensity of choice," measures the agents' sensitivity to changes in forecasting success. Brock and Hommes (1997) focus on the case of large but finite $\alpha$. Branch and Evans (2006a) note that a drawback to finite $\alpha$ is that agents are not fully optimizing. In our earlier paper, it was shown that in a stochastic framework where agents underparameterize their forecasting models, heterogeneity may persist even as $\alpha \rightarrow+\infty$. In the current paper, in the theoretical analysis we again emphasize the case $\alpha \rightarrow+\infty$, which enables us to provide a simple characterization of the possible ME. Then in Section 4, where we examine the system under real-time dynamics, we assume large, finite values of $\alpha$.

There are alternatives to the MNL approach. For example, Cho and Kasa (2006) develop a theory of model selection based on statistical criteria for selecting among possibly misspecified models. An attractive feature of their approach is that it includes a penalty for more complex models. In the current paper, our underparameterized forecasting models are equally complex. However, it would be straightforward to extend our framework to incorporate a third, more complex model and introduce a complexity penalty via a fixed cost in the fitness measure $E u_{j}$. This approach was emphasized by Brock and Hommes (1997) in a deterministic model. It would be interesting then to study whether the simple models can still persist, at least for some time, when there is a more complex and accurate predictor. We leave this issue to future research.

Given that the reduced-form equations governing the competitive equilibrium were derived from utility maximization, it seems natural to ask why we also don't assume that agents choose their forecasting model as the one which maximizes expected lifetime utility. We assume agents minimize their mean square forecast error as a tractable approximation to full utility maximization. We justify this assumption by appealing to bounded rationality: one motivation for the underparameterization is to model agents as econometricians, and thus the choice of forecasting model is a statistical one. As Appendix A shows, the reduced-form AD can be derived from the households' Euler equations, so the choice of forecasting model does not imply the agents are violating their intertemporal optimization conditions. Rather, it is a simplifying assumption that impinges on the firms' pricing decisions. We expect that altering the model so that predictor choice depends on profits will not alter the qualitative results. As we show below, the key property of the model is that there is positive feedback from expectations onto price and this property exists under alternative predictor fitness measures.

One can verify that the MSEs of the predictors imply that

$$
\begin{aligned}
& E u_{1}=\xi_{2}^{2}\left(\rho E z_{1} z_{2}-E z_{2}^{2}\right)-\sigma_{\eta}^{2}, \\
& E u_{2}=\xi_{1}^{2}\left(\tilde{\rho} E z_{1} z_{2}-E z_{1}^{2}\right)-\sigma_{\eta}^{2} .
\end{aligned}
$$


Define the map $F:[0,1] \rightarrow \mathbb{R}$ as

$$
F(n)=\frac{E u_{1}-E u_{2}}{E z_{1 t}^{2}}=\xi_{1}^{2}(1-\rho \tilde{\rho})+\xi_{2}^{2}\left(\rho^{2}-Q\right)
$$

where $Q=E z_{2}^{2} / E z_{1}^{2}$. If condition $\Delta$ is satisfied, $F(\cdot)$ is continuous and well-defined.

Because condition $\Delta$ is satisfied for all $\theta \in[0,1)$, there exists a well-defined mapping $T_{\alpha}$ : $[0,1] \rightarrow[0,1]$ such that $T_{\alpha}=H_{\alpha} \circ F$.

Definition. A Misspecification Equilibrium (ME) is a fixed point, $n^{*}$, of $T_{\alpha}$.

In a Misspecification Equilibrium the forecast parameters satisfy the orthogonality condition and the predictor proportions are determined by the MNL. In equilibrium, they are, therefore, both endogenously determined.

Proposition 2. A Misspecification Equilibrium exists.

This result follows since $T_{\alpha}:[0,1] \rightarrow[0,1]$ is continuous and Brouwer's theorem ensures that a fixed point exists. ${ }^{12}$ By developing details of the map $F$ we are able to investigate further the set of ME.

Proposition 3. The function $F(n)$ is monotonically increasing for all $0 \leqslant \theta<1$.

Appendix A sketches the proofs to all propositions. Theoretical details that carry over from the cobweb model can be found in Branch and Evans (2006a).

From the equation for expected utility it can be shown that

$$
\begin{array}{ll}
F(1) \gtrless 0 & \text { iff }(1-\rho \tilde{\rho}) \xi_{1}^{2}(1) \gtrless\left(Q-\rho^{2}\right) \xi_{2}^{2}(1), \\
F(0) \gtrless 0 & \text { iff }(1-\rho \tilde{\rho}) \xi_{1}^{2}(0) \gtrless\left(Q-\rho^{2}\right) \xi_{2}^{2}(0)
\end{array}
$$

where $Q=\frac{E z_{2}^{2}}{E z_{1}^{2}}$. Furthermore, from (7) we have

$$
\begin{aligned}
& \frac{\left(\xi_{1}(1)\right)^{2}}{\left(\xi_{2}(1)\right)^{2}}=\frac{\left(\left(\gamma_{1} a_{11}+\gamma_{2} a_{21}\right)+\left(\gamma_{1} a_{12}+\gamma_{2} a_{22}\right) \theta \rho\right)^{2}}{(1-\theta)^{2}\left(\gamma_{1} a_{12}+\gamma_{2} a_{22}\right)^{2}} \equiv B_{1}, \\
& \frac{\left(\xi_{1}(0)\right)^{2}}{\left(\xi_{2}(0)\right)}=\frac{\left(\gamma_{1} a_{11}+\gamma_{2} a_{21}\right)^{2}(1-\theta)^{2}}{\left(\left(\gamma_{1} a_{11}+\gamma_{2} a_{21}\right) \theta \tilde{\rho}+\gamma_{1} a_{12}+\gamma_{2} a_{22}\right)^{2}} \equiv B_{0} .
\end{aligned}
$$

Note that $0<B_{0}<B_{1}$. Recall that $Q, \rho$, and $\tilde{\rho}$ are determined by $A$ and $\Sigma_{\varepsilon}$. The above results and Proposition 3 imply:

Lemma 4. There are three possible cases depending on $A, \theta, \gamma$ and $\Sigma_{\epsilon}$ :

(1) Condition PM: $F(0)<0$ and $F(1)>0$. Condition PM is satisfied when $(1-\rho \tilde{\rho}) B_{0}+\rho^{2}<$ $Q<(1-\rho \tilde{\rho}) B_{1}+\rho^{2}$.

12 Branch and Evans (2006a) prove existence of a Misspecification Equilibrium for an $n$-dimensional vector $z_{t}$ following a stationary $\operatorname{VAR}(p)$ process, and an arbitrary list of misspecified models, provided $|\theta|$ is sufficiently small. The proof in Branch-Evans does not rely on the sign of $\theta$. 
(2) Condition P1: $F(0)>0$ and $F(1)>0$. Condition $P 1$ arises when $Q<(1-\rho \tilde{\rho}) B_{0}+\rho^{2}$.

(3) Condition PO: $F(0)<0$ and $F(1)<0$. Condition PO arises when $Q>(1-\rho \tilde{\rho}) B_{1}+\rho^{2}$.

Remark. $\rho \tilde{\rho}=1$ is ruled out by the positive definiteness of $\Sigma_{\varepsilon}$.

Below we give numerical examples of when each condition may arise.

Under Condition PM, $F(0)<0$ and $F(1)>0$ implies that either model is preferred so long as all agents coordinate on that model; thus, there is no incentive for agents to deviate from homogeneity. When Condition P1 or P0 holds one model always dominates the other.

Lemma 4 allows for a characterization of the set of Misspecification Equilibria for large $\alpha$. Let

$$
N_{\alpha}=\left\{n^{*} \mid T_{\alpha}\left(n^{*}\right)=n^{*}\right\} .
$$

We now present our primary existence result for large $\alpha$.

Proposition 5. Characterization of Misspecification Equilibria for large $\alpha$ :

(1) Under Condition PM, as $\alpha \rightarrow \infty, N_{\alpha} \rightarrow\{0, \hat{n}, 1\}$ where $\hat{n}_{1}$ is s.t. $F\left(\hat{n}_{1}\right)=0$.

(2) Under Condition PO, as $\alpha \rightarrow \infty, N_{\alpha} \rightarrow\{0\}$.

(3) Under Condition P1, as $\alpha \rightarrow \infty, N_{\alpha} \rightarrow\{1\}$.

The remainder of the paper is primarily concerned with Case 1 in which there are multiple equilibria. It should be briefly noted that an ME does not coincide with the unique REE in (2). For all $n^{*} \in N_{\alpha}$ a comparison of (2) and the ME in (6) and (7) (for a given $n^{*}$ ) shows that the ME has different relative weights on the exogenous variables. ${ }^{13}$ Interestingly, there may exist multiple ME even though there is a unique REE. The 'instability' that results from misspecification is key for generating endogenous regime change in the Lucas model.

\subsection{Further intuition for multiple equilibria}

The existence of multiple equilibria, and the resulting real-time learning and dynamic predictor selection dynamics, are key results. In this regard, greater intuition of when multiple equilibria arises is useful. The existence of multiple equilibria, as demonstrated by Proposition 5, depends on the asymptotic properties of the $z$ process, and on both the direct $\left(\gamma^{\prime} A\right)$ and indirect $(\theta)$ effect of $z$ on inflation. This subsection presents the intuition on the relationship between the direct and indirect effects.

For ease of exposition, assume $\rho=\tilde{\rho}=0$. From Proposition 5 multiple ME arise when

$$
B_{0}<Q<B_{1},
$$

where

$$
B_{1}=\frac{\left(\gamma_{1} a_{11}+\gamma_{2} a_{21}\right)^{2}}{(1-\theta)^{2}\left(\gamma_{1} a_{12}+\gamma_{2} a_{22}\right)^{2}},
$$

13 Adam (2005a) considers a New Keynesian model where agents are restricted to univariate forecasting models. In his model, though, there exist equilibria which are REE. Guse (2005) also studies a model with multiple REE and where agents' forecasting models are distributed across the representations consistent with each REE. 


$$
B_{0}=\frac{\left(\gamma a_{11}+\gamma_{2} a_{21}\right)^{2}(1-\theta)^{2}}{\left(\gamma_{1} a_{12}+\gamma_{2} a_{22}\right)^{2}} .
$$

Now suppose that $\theta=0$. Then $B_{0}=B_{1}$ and there does not exist any $Q$, hence any $z$, such that multiple equilibria exist. Instead suppose that $\theta \rightarrow 1$. Then $B_{0} \rightarrow 0$ and $B_{1} \rightarrow \infty$. In this instance, the entire range of uncorrelated, bivariate $\operatorname{VAR}(1)$ will lead to multiple equilibria.

The condition PM places restrictions on the interaction between the direct and indirect effects of the model. When there is no feedback from expectations onto the state, then it is clear that agents will always choose the predictor with the highest direct effect. When the self-referential parameter is high, then the indirect effect magnifies the direct effect of the components of $z$. In these instances it is possible that coordination on a particular forecasting model will produce an indirect effect sufficiently stronger than the direct effect so that this predictor dominates in expected MSE. Significantly, the existence of multiple equilibria arises from the coordinating forces of positive feedback.

\subsection{Numerical examples}

We turn now to a numerical illustration. Figure 2 gives the T-maps for various values of $\alpha$. The upper part of the figure shows the T-maps corresponding to (starting from $n=0$ and moving clockwise) $\alpha=10, \alpha=20, \alpha=50, \alpha=1000$. We set

$$
A=\left[\begin{array}{cc}
0.5 & 0.001 \\
0.001 & 0.3
\end{array}\right]
$$

with $\gamma^{\prime}=[0.5,0.75]$,

$$
\Sigma_{\varepsilon}=\left[\begin{array}{cc}
0.03 & 0.001 \\
0.001 & 0.15
\end{array}\right]
$$

and $\theta=0.6$. The bottom portion of the figure is the profit difference function $F(n)$.

The matrix $A, \Sigma_{\varepsilon}$, and $\theta$ have been chosen so that Condition PM holds. Condition PM holds under many other parameterizations as discussed above. We chose these parameters as they deliver quantitatively reasonable results in the section on real-time learning and dynamic predictor selection.

A key property of the model is that as $\alpha \rightarrow \infty$

$$
H_{\alpha}(x) \rightarrow \begin{cases}1 & \text { if } x>0, \\ 0 & \text { if } x<0, \\ 1 / 2 & \text { if } x=0\end{cases}
$$

and this governs the behavior of $T_{\alpha}=H_{\alpha} \circ F$. Since $H_{\alpha}$ is an increasing function and $F$ is monotonically increasing, it follows that $T_{\alpha}$ is increasing. Under Condition PM it is clear that (9) implies existence of three fixed points for $\alpha$ sufficiently large. The figure illustrates this intuition.

This example makes it clear that multiple equilibria can exist in the Lucas-type monetary model. When agents underparameterize there is an incentive to coordinate on a particular forecasting model. Interestingly, though, there also exists an interior equilibrium. Below we show that this equilibrium is unstable under learning. The existence of multiple ME suggests there may be interesting learning phenomena in the model. We take up this issue in the section below.

The particular parameterization which leads to this figure produces the following asymptotic covariance matrix for $z_{t}$ :

$$
\Sigma_{z}=\left[\begin{array}{cc}
0.04 & 0.0013 \\
0.0013 & 0.1648
\end{array}\right] .
$$



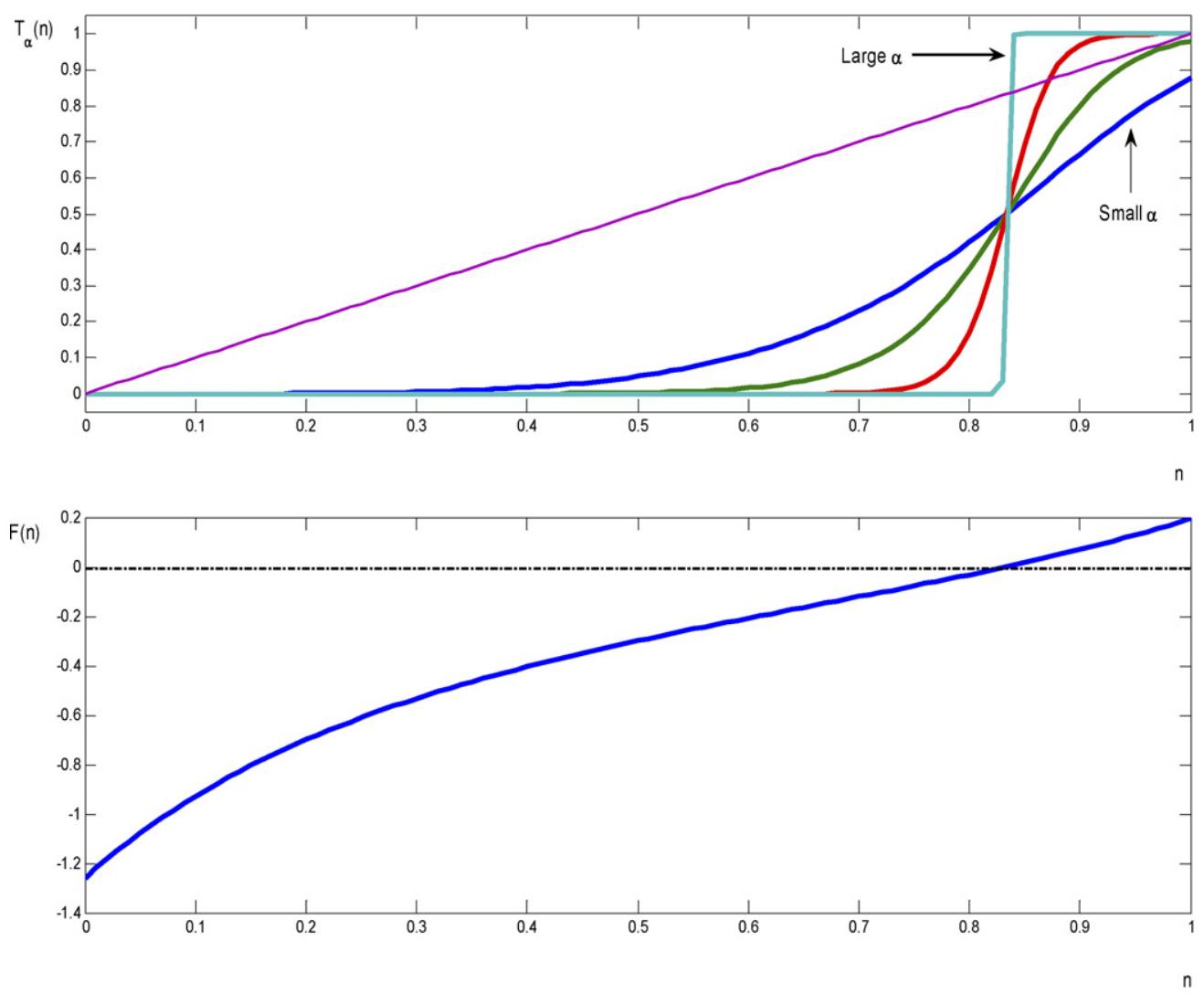

Fig. 2. T-map for various values of $\alpha$ and $\theta=0.60$.

Notice that the variance of $z_{2}$ is approximately 4 times that of $z_{1}$. The effect of this can be seen in Fig. 2 where the 'basin of attraction' for the $n=0 \mathrm{ME}$ is larger than for the $n=1 \mathrm{ME}$. A priori we would expect a real-time version of this economy to spend, on average, more time near $n=0$ than $n=1$. This logic will be key in Section 5 .

It should be emphasized that in other contexts there may exist a unique interior ME. Branch and Evans (2006a) illustrate this case by developing the framework in the context of the cobweb model. The existence of an ME with heterogeneity—what Branch and Evans (2006a) call Intrinsic Heterogeneity-exists for precisely the opposite reasoning for multiple $\mathrm{ME}$ in the Lucas model. In the cobweb model there is negative feedback from expectations onto the state. Under certain conditions there is an incentive for agents to deviate from the consensus model. Thus, the equilibrium forces push agents away from homogeneity. In the Lucas model the equilibrium forces, as a result of the positive feedback, push the economy towards homogeneity. These results illustrate the multiplicity of equilibrium phenomena that can arise depending on the self-referential features of a simple model.

\section{Learning and dynamic predictor selection}

In this section we first address whether the Misspecification Equilibria are attainable under real-time econometric learning and dynamic predictor selection. We now replace optimal linear 
projections with real-time estimates formed via recursive least squares (RLS). We also assume that agents choose their model each period based on an estimate of mean square error. In Section 5 we replace RLS with a constant gain updating rule of the form used, for example, in Evans and Honkapohja (1993), Sargent (1999) and Cho et al. (2002), and we also employ a constant gain version of the dynamic predictor selection introduced by Brock and Hommes (1997).

We thus replace the equilibrium stochastic process (6) with one that has time-varying beliefs and predictor proportions. Below we provide details on how the key relationships are altered. This section briefly discusses the stability of the equilibrium under recursive least squares. We model least squares learning as in Branch and Evans (2006a). Agents have a RLS updating rule with which they form estimates of the belief parameters $b_{t}^{1}, b_{t}^{2}$. They also estimate the MSE of each predictor by constructing a moving average of past squared forecast errors with equal weight given to all time periods. Given estimates for the belief parameters and predictor fitness, agents choose their forecasting model according to the MNL map in real time. ${ }^{14}$

We now assume the equilibrium stochastic process is given by

$$
\pi_{t}=\xi_{1}\left(b_{t-1}^{1}, n_{1, t-1}\right) z_{1, t-1}+\xi_{2}\left(b_{t-1}^{2}, n_{1, t-1}\right) z_{2, t-1}+\eta_{t} .
$$

Agents use a recursive least squares updating rule,

$$
b_{t}^{j}=b_{t-1}^{j}+\kappa_{t} R_{j, t}^{-1} z_{j, t-1}\left(\pi_{t}-b_{t-1}^{j} z_{j, t-1}\right), \quad j=1,2 .
$$

where

$$
R_{j, t}=R_{j, t-1}+\kappa_{t}\left(z_{j, t-1}^{2}-R_{j, t-1}\right), \quad j=1,2 .
$$

We consider two possible cases for the gain sequence $\kappa_{t}$ : under decreasing gain, $\kappa_{t}=t^{-1}$ so that $\kappa_{t} \rightarrow 0$; under constant gain, $\kappa_{t}=\kappa \in(0,1)$.

We also assume agents recursively update mean-square forecast error according to

$$
M S E_{j, t}=M S E_{j, t-1}+\lambda_{t}\left(\left(\pi_{t}-\pi_{j, t}^{e}\right)^{2}-M S E_{j, t-1}\right), \quad j=1,2 .
$$

We again consider two possible cases for the gain sequence $\lambda_{t}$ : under decreasing gain, $\lambda_{t}=t^{-1}$ so that $\lambda_{t} \rightarrow 0$; under constant gain, $\lambda_{t}=\lambda \in(0,1)$.

We first look at the case of decreasing gain for both $\kappa_{t}, \lambda_{t}$. We then turn in the next Section to our main emphasis of constant gain updating.

\subsection{Stability under decreasing gain}

In this subsection we study whether the sequence of estimates $b_{t}^{1}, b_{t}^{2}$ and predictor proportions $n_{1, t}$ converge to a Misspecification Equilibrium. ${ }^{15}$ Our aim is to use simulations to ascertain which equilibria are stable under real-time learning and dynamic predictor selection. Establishing analytical convergence is beyond the scope of this paper.

We continue with the parameterization in the previous section which yielded multiple ME. We set

$$
A=\left[\begin{array}{cc}
0.5 & 0.001 \\
0.001 & 0.3
\end{array}\right], \quad \Sigma_{\varepsilon}=\left[\begin{array}{cc}
0.03 & 0.001 \\
0.001 & 0.15
\end{array}\right]
$$

\footnotetext{
14 A point made in Branch and Evans (2006a) is that stability of a steady-state depends on how more recent forecast errors are weighted in the moving average calculation. In particular, as the most recent error is weighted more heavily then instability will result as in Brock and Hommes (1997, 1998).

15 Because the analysis is numerical we are being deliberately vague in what sense these sequences converge.
} 
and $\gamma^{\prime}=[0.5,0.75]$. We also set $\theta=0.6$ and $\alpha=1000 .{ }^{16}$ We simulate the model for 5,000 time periods. The initial value of the VAR is equal to a realization of its white noise shock, i.e., $z_{0}=\varepsilon_{0}$. The initial value $n_{1,0}$ is drawn from a uniform distribution on $[0,1]$ and $b_{j, 0}, j=1,2$ is drawn from a uniform distribution on [0,2]. The initial estimated variances are set $R_{1,0}=R_{2,0}=1$.

Figure 3 illustrates the results of two representative simulations. The top panel plots the simulated proportions $n_{t}$ against time. Recall that for the chosen parameters there exist three equilibria. The plot demonstrates that only the equilibria with homogeneous expectations are stable under learning and dynamic predictor selection. The dynamics quickly converge to either $n=0$ or $n=1$. The bottom panel plots the reduced form equilibrium parameters $b_{t-1}^{1}, b_{t-1}^{2}$. In each panel there are two horizontal lines which correspond to the parameter values in either the $n=0$ or $n=1 \mathrm{ME}$. In the $b^{1}$ panel the top horizontal line corresponds to the $n=1$ equilibrium and in the $b^{2}$ panel the top horizontal line is for the $n=0$ equilibrium. As seen in the top panel,
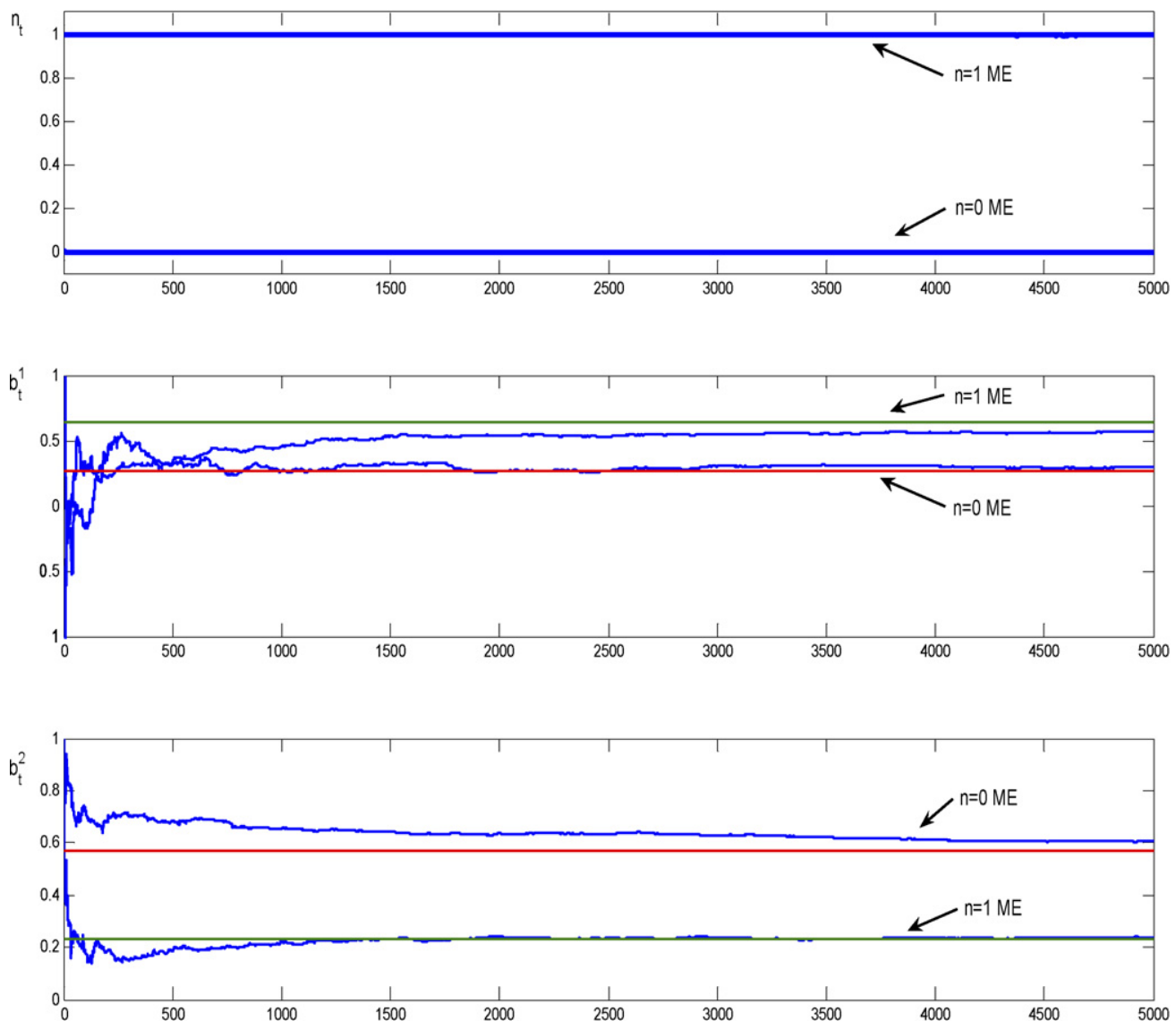

Fig. 3. Two RLS learning and dynamic predictor selection trajectories converging to ME. Note: horizontal lines correspond to equilibrium parameter values.

16 Similar results were obtained for other parameter settings. The speed of convergence is sensitive to larger values of $\theta$ and $\alpha$. 
these parameters converge to their ME values. Which equilibrium the dynamics converge to depends on the basins of attraction. As we emphasize in the next section, these basins are sensitive to the parameterization of the $z_{t}$ process. Thus, we conclude that ME with $n \in\{0,1\}$ are locally stable under learning and dynamic predictor selection.

The intuition for this stability is as follows. The multiple equilibria results from an incentive for agents to coordinate on a single model. These coordinating forces render the interior equilibrium, with heterogeneity, unstable under learning. Suppose the dynamics begin in a neighborhood of the interior equilibrium. Because the profit function is monotonically increasing, as more agents mass onto a particular model then more agents will also want to use that model. The dynamics are repelled from the neighborhood of the interior steady-state and towards one of the other ME. To which ME the dynamics converge depends on the basin of attraction in which the initial conditions lie.

This result is, again, distinct from the result in Branch and Evans (2006a). In that paper, there is a unique Misspecification Equilibrium which is stable under learning. In the current paper we have multiple equilibria on the boundary of the unit interval that are locally stable under learning. This result leads to interesting dynamics when agents update with a constant gain learning algorithm.

\section{Real-time learning with constant gain}

It has been suggested by Sargent (1999), among others, that agents concerned with structural change should use a constant gain version of RLS to generate parameter estimates. A constant gain algorithm involves a time-invariant gain which places a high relative weight on recent versus distant outcomes. If agents are concerned about structural change then a constant gain algorithm will better pick up a change in parameters. It has also been argued by Orphanides and Williams (2005a) that constant gain learning is more reasonable than RLS learning because the learning rule itself is stationary whereas it is time-dependent in RLS. Empirical support for constant gain learning is provided in Orphanides and Williams (2005b), Branch and Evans (2006b), and Milani (2005).

In the Lucas model with misspecification we showed that there may exist multiple equilibria. Moreover, a subset of these equilibria are stable under learning with a decreasing gain algorithm such as RLS. In these equilibria there is an incentive for agents to coordinate on the same forecasting model. If a large enough proportion of agents suddenly switch forecasting models then the economy will switch from one stable ME to another. Agents concerned with this possibility should use a constant gain algorithm instead of a decreasing gain to account for possible regime change.

There has been an explosion in research adopting constant gain learning rules. Examples include Bullard and Cho (2005), Cho and Kasa (2003), Cho et al. (2002), Evans and Honkapohja (1993, 2001), Evans and Ramey (2006), Kasa (2004), Orphanides and Williams (2005a), Sargent (1999), Sargent and Williams (2005) and Williams (2004a, 2004b). In many of these models constant gain learning can lead to abrupt changes or 'escapes' in the dynamics. For example, in models with multiple equilibria, such as Evans and Honkapohja (1993, 2001), occasional shocks can lead agents to believe the economy has shifted to a new equilibrium. The result of these beliefs is a self-confirming shift to the new equilibrium. Unlike sunspot equilibria, these shifts are driven entirely by agents' recursive parameter estimates. In Sargent (1999), Cho and Kasa (2003), Cho et al. (2002), Bullard and Cho (2005), and McGough (2006), occasional large shocks can lead to temporary deviations from the equilibrium that is uniquely stable under RLS. 
The same logic underlying the use of constant gain RLS for parameter estimation carries over to the estimate of the relative fitness of the two forecast rules. Agents who are concerned about structural change, including shifts taking the form of occasional regime changes, would want to allow for the possibility that the better performing forecast rule may shift over time. In order to remain alert to such shifts, agents would weight recent forecast errors more heavily than past forecast errors when computing the average mean square error of each rule. This is equivalent to a constant gain estimate of the average mean square error and leads to dynamic predictor selection following a stochastic process.

In this section we examine the implications of constant gain learning and dynamic predictor selection in the Lucas model with multiple misspecification equilibria. Note, though, that we expect to find distinct dynamics from the studies listed above. This is because in each misspecification equilibrium the mean inflation rate, and hence mean output, is the same. Instead the variance of inflation differs across equilibria. We show that endogenous inflation and output volatility arise through two channels: (1) the drift in beliefs from parameter learning with a constant gain RLS; (2) dynamic predictor selection with a geometric average of past squared forecast errors.

Our results show that this combination potentially could generate observed volatility along the lines presented in Section 2. ${ }^{17}$ In Branch and Evans (2006a) the joint learning of parameters and dynamic predictor selection was presented as a novel extension of Evans and Honkapohja (2001) and Brock and Hommes (1997), but that model possesses a unique equilibrium and the focus was on heterogeneity and stability. Here the focus is on endogenous volatility resulting from the dual learning process in a set-up with multiple equilibria.

\subsection{Joint learning with constant gain algorithms}

With constant gains $\kappa_{t}=\kappa>0, \lambda_{t}=\lambda>0$ the dynamics will not converge to a Misspecification Equilibrium. However, we note that because the ME with $n \in\{0,1\}$ are stable under decreasing gain learning, we anticipate that the dynamics will spend a considerable portion of their time in a neighborhood of the stable MEs.

Under constant gain, $M S E_{j, t}$ estimates the MSE as an average of past squared forecast errors with weights declining geometrically at rate $1-\lambda$. Similarly, constant gain least squares aims to minimize a weighted sum of squared errors where the weight declines geometrically at rate $1-\kappa$. In choosing $\kappa, \lambda$ there is a trade-off in tracking structural change versus filtering noise. How strongly $n_{j, t}$ and $b_{j, t}$ react to these shocks then depends on $\lambda, \kappa$, the 'intensity of choice' parameter in the MNL mapping $\alpha$, and the relative size of the basins of attraction of the two stable steady-state ME. Switching as a result of changes in relative MSE is the second source of endogenous volatility. Suitable choices of $\lambda, \kappa, \alpha$ determine the degree to which the model exhibits parameter drift and/or endogenous switching between basins of attraction.

For simplicity, our model does not explicitly incorporate structural change. However, the switches between equilibria provide a (self-fulfilling) rationale for the use of constant rather than decreasing gains. Evans and Honkapohja (1993) construct a model with multiple stable equilibria and pin down the optimal constant gain in agents' learning algorithm as an approximate Nash equilibrium. In these settings, there is an optimal gain because agents are alert to

17 Bacchetta and Van Wincoop (2004) develop a model in which agents have private signals and underparameterized forecasting models. They show that the predictive power of certain macroeconomic fundamentals may change over time as agents' higher order expectations cause them to occasionally over-react to news. 
potential switches between equilibria. An extension of the present paper along these lines would be interesting and is left to future research.

As a means of illustrating the intuition we first present a simulation from a parameterization designed to yield striking results. We first let the asymptotic moments of the $z$ process differ markedly. Set

$$
A=\left[\begin{array}{cc}
0.5 & 0.001 \\
0.001 & 0.3
\end{array}\right], \quad \Sigma_{\varepsilon}=\left[\begin{array}{ll}
0.2 & 0.1 \\
0.1 & 3.2
\end{array}\right],
$$

$\gamma^{\prime}=[0.5,0.5], \theta=0.95$, and $\alpha=1000$. We set $\kappa=0.15$ and $\lambda=0.35$. With this parameterization the asymptotic covariance matrix for $z$ is

$$
\Sigma_{z}=\left[\begin{array}{ll}
0.2668 & 0.1190 \\
0.1190 & 3.5166
\end{array}\right] .
$$

Figures 4-5 illustrate typical trajectories when $\alpha=1000,10$ respectively. Figure 4 illustrates a number of switches between equilibria during the period 1000-2500. Notice that in this plot the system spends most of its time at the $n=0 \mathrm{ME}$. Moreover, when the dynamics switch to the $n=1 \mathrm{ME}$ it is for relatively short periods. This is because the basin of attraction for $n=0$ is relatively large, and it takes a greater accumulation of shocks to place the economy in the $n=1$ basin. This parameterization was designed to make the volatility differences dramatic. In so doing we set the variance of inflation at the $n=0 \mathrm{ME}$ much greater than at the $n=1 \mathrm{ME}$. Because the variance of $z_{2}$ is much greater than $z_{1}$ and $z_{1}, z_{2}$ are weakly correlated, the basin of attraction for the 'lower' ME is larger. It is only when a very large proportion of agents use the $z_{1}$ forecasting model is it in the best interests of all agents to use that model. Notice also that these dynamics exist for both large and small values of $\alpha$.
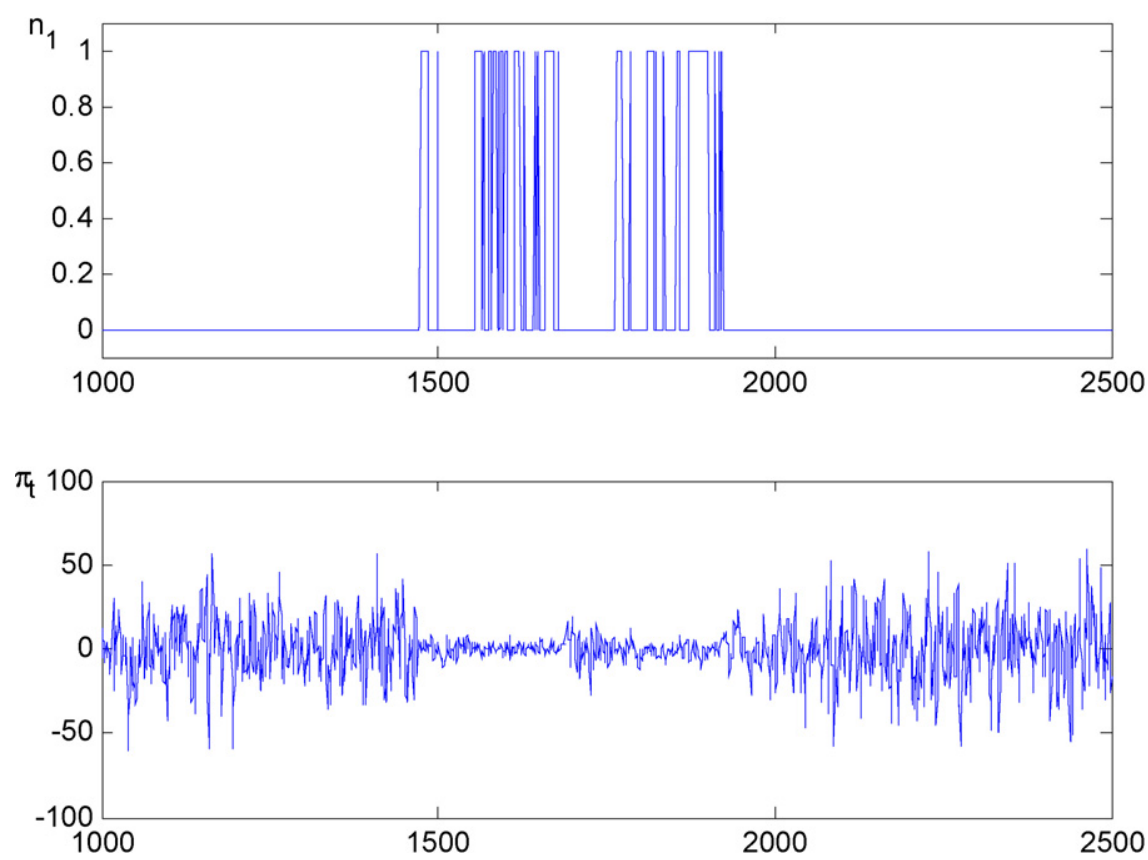

Fig. 4. Constant gain learning with $\theta=0.95, \kappa=0.15, \lambda=0.35, \alpha=1000$. 

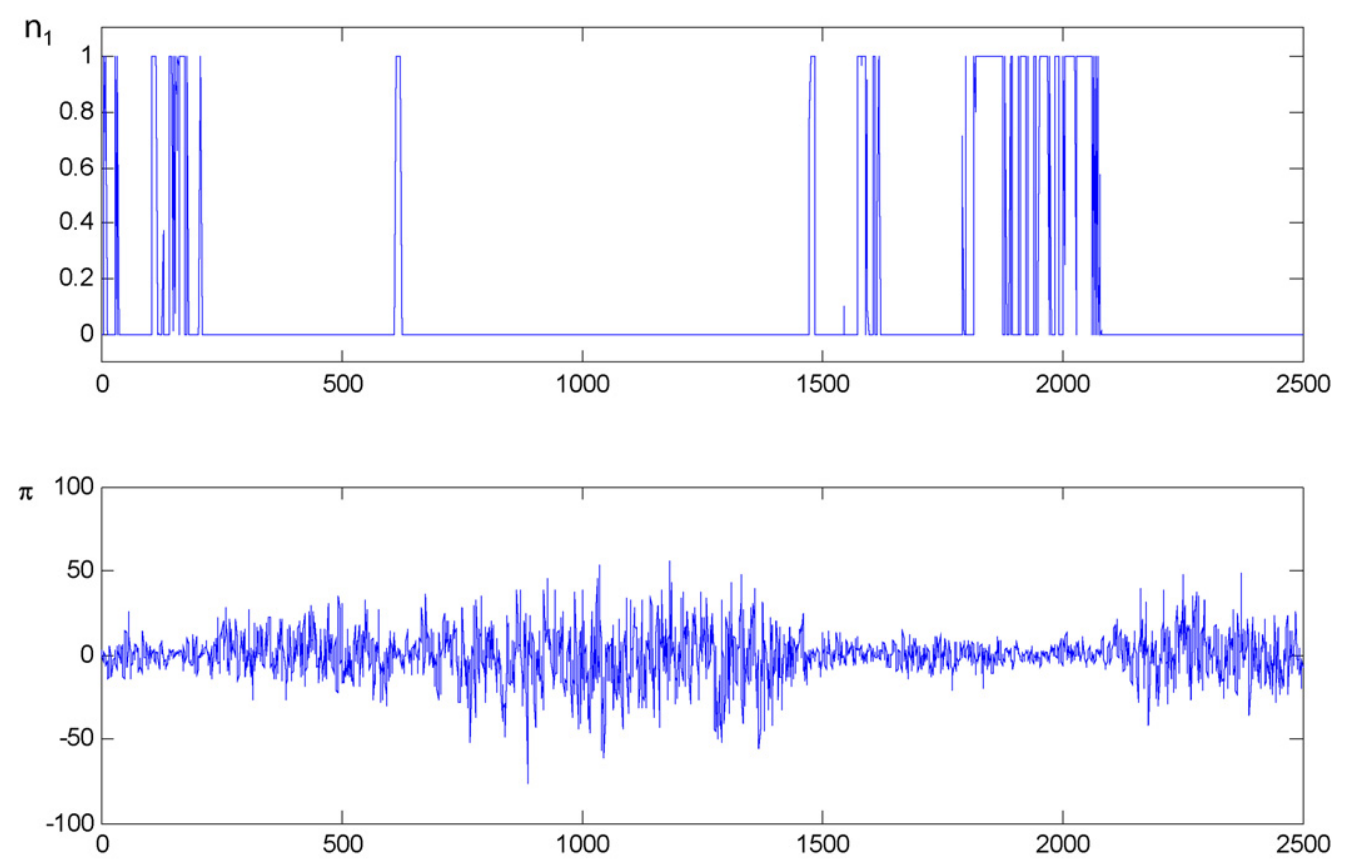

Fig. 5. Constant gain learning with $\theta=0.95, \kappa=0.15, \lambda=0.35, \alpha=10$.

Once a switch takes place there is considerable differences in inflation volatility. Notice that during the periods of frequent switches between ME—-so that, on average, more time is spent at the $n=1 \mathrm{ME}$ - the inflation volatility switches between a high rate and a low rate. In the $n=1$ $\mathrm{ME}$ there is no positive feedback from $z_{2}$ through expectations onto the inflation rate. Thus, in the $n=1 \mathrm{ME}$ a larger relative weight is placed on $z_{1}$ which is a random variable with a lower asymptotic variance. Hence, we see much lower inflation variances.

These simulations suggest an interpretation to the empirical regularity discussed in the beginning of the paper. In the Lucas model with model underparameterization there may exist multiple equilibria where agents ignore some relevant information when forecasting inflation. If there are significant differences between the information they incorporate and ignore, then the expectational feedback will make the inflation variances differ across these equilibria. To explore this hypothesis further we parameterize the model so that unconditional variances have plausible magnitudes. We also seek to isolate the contributions of parameter learning and mean-square error learning to the endogenous volatility.

We now set the parameters as in Section 3.4 and Section 4, which we reproduce here for convenience:

$$
A=\left[\begin{array}{cc}
0.5 & 0.001 \\
0.001 & 0.3
\end{array}\right], \quad \Sigma_{\varepsilon}=\left[\begin{array}{cc}
0.03 & 0.001 \\
0.001 & 0.15
\end{array}\right]
$$

with $\theta=0.6, \gamma^{\prime}=(0.5,0.75) \kappa=0.01, \lambda=0.04$. A value of $\kappa=0.01$ in monthly data is consistent with Branch and Evans (2006b). There is not a consensus in the literature on the appropriate value of $\lambda$; to remain neutral on the issue, we present below simulations with low (0.01) and high (0.20) values as well. We simulate the model, first with a transient period of length 15,000, and then for 5000 periods in which we report the results in the figures below. 
To isolate the effect of parameter drift versus dual learning our strategy is as follows. We first present results where we fix the proportion of agents $n$ to one of its ME values, but allow agents to update their parameters with constant gain least squares. This is analogous to the approach pursued, for example, by Orphanides and Williams (2005a) in a full-information setting. We then present simulations with dual learning.

Figure 6 presents the results from a typical simulation. There are 5 panels in the figure. Beginning from the northwest and moving clockwise they are: predictor proportion $n$, belief parameters $b_{t}^{1}, b_{t}^{2}$, time $t$ estimated unconditional variance of output and price respectively. The unconditional variances are computed as moving averages with window length 200 of the variance of the simulated time series. We set $n=0$, though similar results obtain if we instead set $n=1$. The horizontal lines in the figure are the ME values.

Figure 6 shows that some of the endogenous volatility can be attributed to parameter drift. With a constant gain in the least-squares algorithm agents are sensitive to structural change. This is why in the two panels on the right-hand side of the figure there is considerable parameter drift. This parameter drift manifests itself in the reduced-form parameters of the model and induces
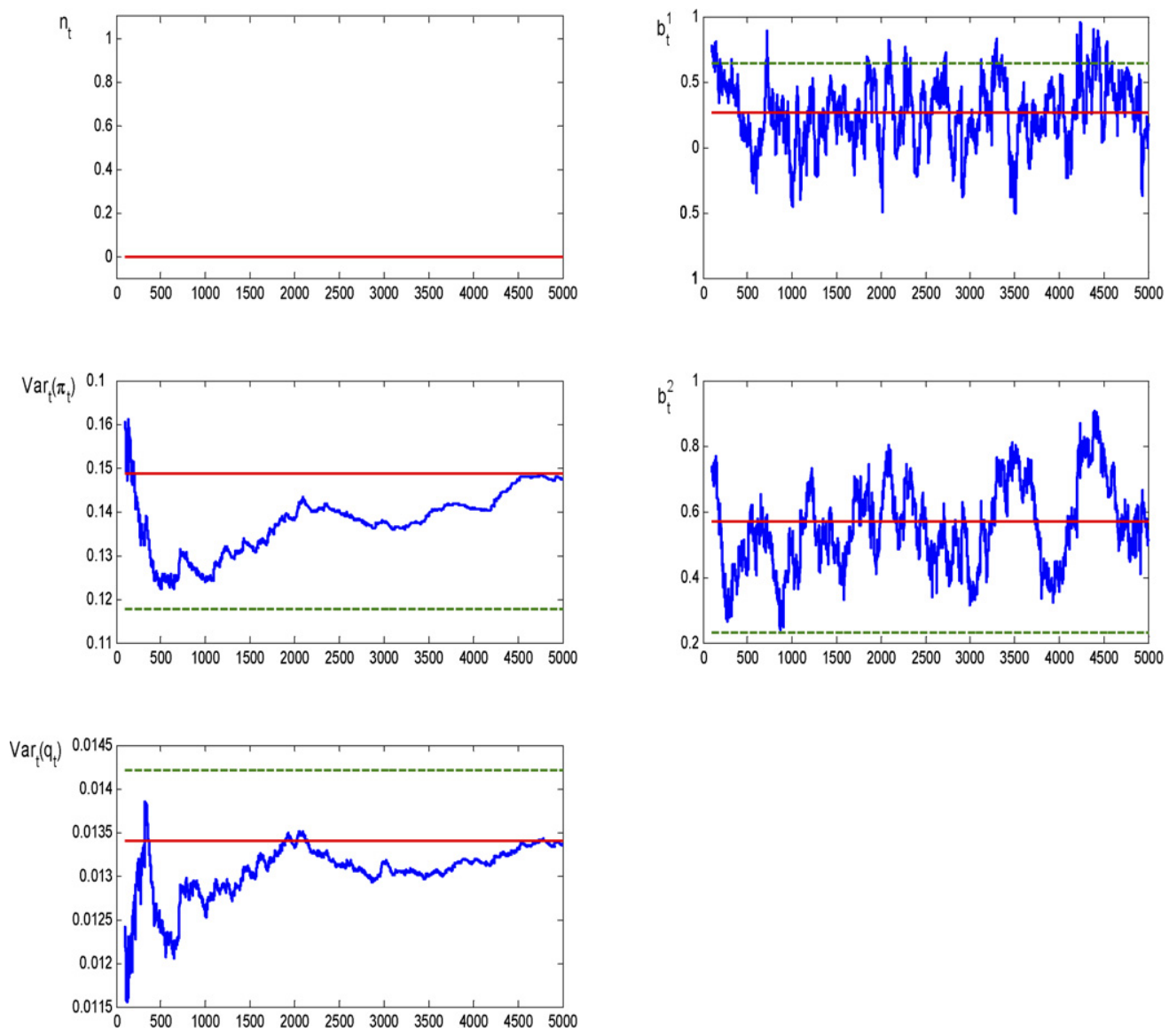

Fig. 6. Parameter learning and no dynamic predictor selection with $n=0$. Solid line is $n=0 \mathrm{ME}$ and dashed line represents $n=1 \mathrm{ME}$. 
some endogenous volatility. However, it does not generate the type of regime-shifting volatility that was documented in Section 2 and elsewhere in the literature.

Figure 7 now puts both elements together to illustrate that dual learning can account for endogenous volatility. Figure 7 demonstrates that combining parameter drift and dynamic predictor selection induces a stochastic process for inflation and output with volatility which both drifts and switches between high and low volatility regimes.

The length of time spent in a neighborhood of an ME depends in a complicated way on the size of the basin of attraction, the gains $\kappa, \lambda$, and the intensity of choice $\alpha$. Figure 8 presents a 'closeup' view of a particular segment of the simulation in Fig. 7. This segment clearly demonstrates the drifting and regime switching inflation and output volatility.

To explore further how the choice of $\lambda$ impacts the switching between equilibria and the resulting drift and/or regime switching volatility, we present two more simulations with low and high values of $\lambda$. Figure 9 presents a simulation with the same parameter values except now $\lambda=0.20$, while Figure 10 sets $\lambda=0.01$. Figure 9 clearly demonstrates that the frequency of switching between equilibria, and the resulting regime switching variances, increases with $\lambda$ the parameter governing the weighted average of past forecast errors. For sufficiently small $\lambda$, there is no endogenous switching between equilibria and the time-series are mostly characterized by volatility drift. Thus, depending on the appropriate value of $\lambda$ or $\kappa$, a serious calibration
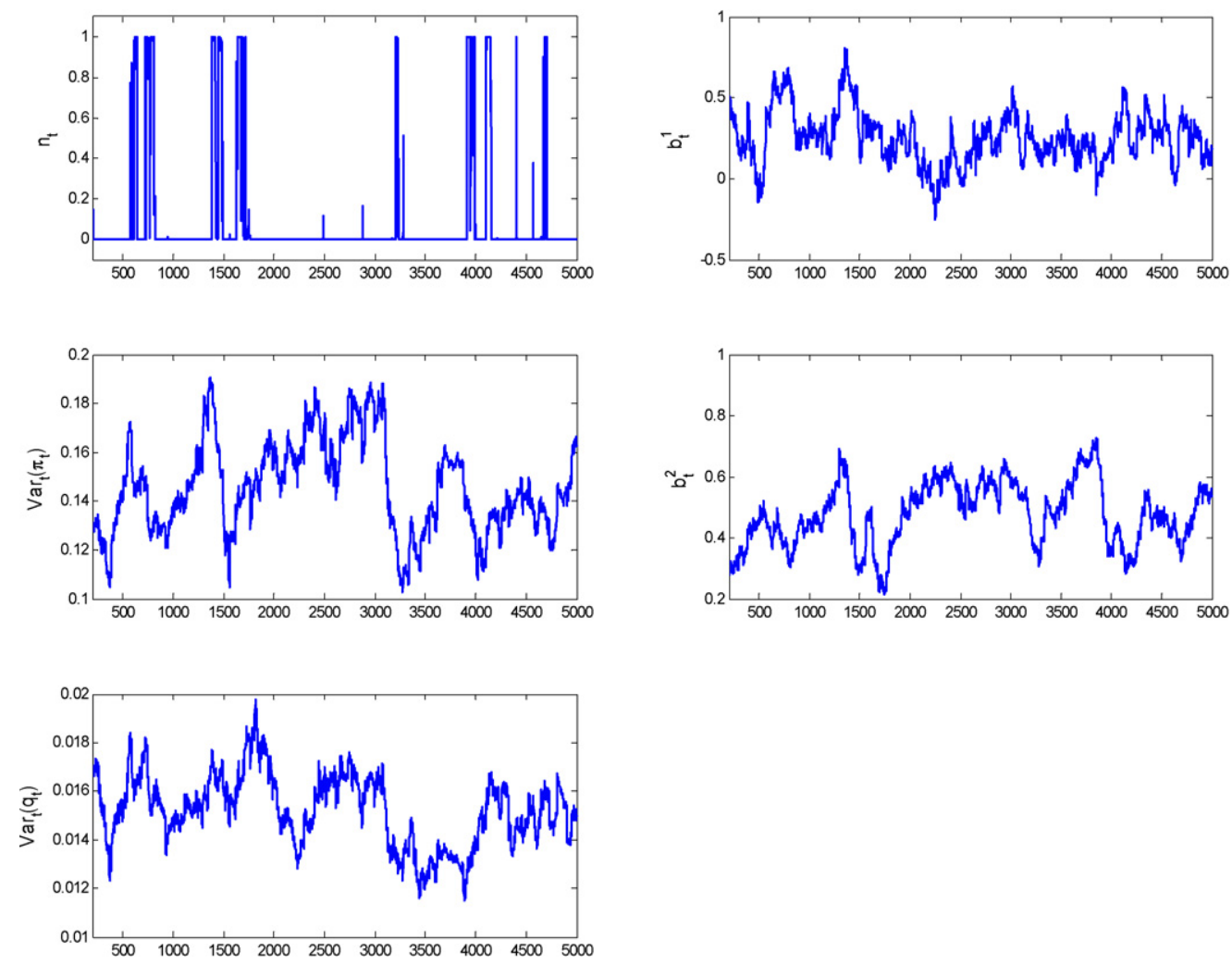

Fig. 7. Parameter learning and dynamic predictor selection $(\lambda=0.04)$. 

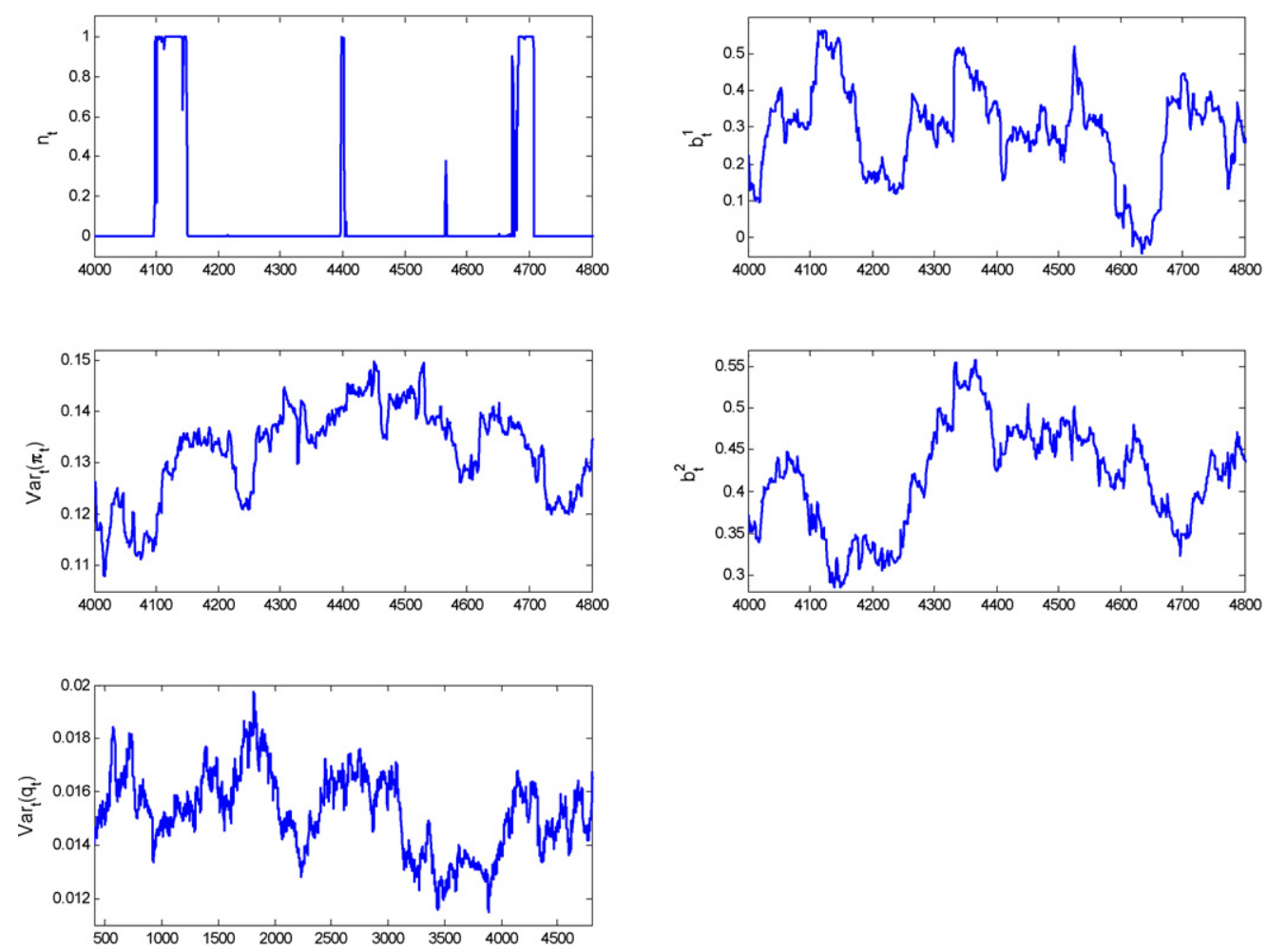

Fig. 8. Close-up of Fig. 7.

exercise might be able to shed light on some of the issues raised by Sims-Zha and CogleySargent.

The above results lead to drifting and regime switching volatility as agents switch between underparameterized forecasting models. Recent research by Cogley and Sargent (2005b) and Brock et al. (2003) instead take a Bayesian model averaging approach. It is not obvious whether it would still be possible to generate heterogeneity and model switching if model averaging were available as a third alternative. The existence of heterogeneity is an important issue, and it would be interesting for future research to study whether heterogeneous expectations will still arise when agents have the option to do Bayesian model averaging.

\subsection{Further discussion}

As a means of further discussion, an overview is helpful. We take a business cycle model where unexpected shocks drive real output fluctuations. We assume bounded rationality but preserve the spirit of Muth's hypothesis and find that there exist multiple equilibria in a model with a unique REE. Moreover, these multiple equilibria arise because the self-referential feature of the Lucas model provides an incentive for agents to forecast with the same model. Each equilibrium can be characterized by the forecasting model that generates it, and each predictor produces distinct forecasts. For practical purposes, the important theoretical implication of the multiple equilibria result is that the self-referential property alters the effects the exogenous stochastic processes have on inflation and output; the positive feedback from expectations onto inflation 

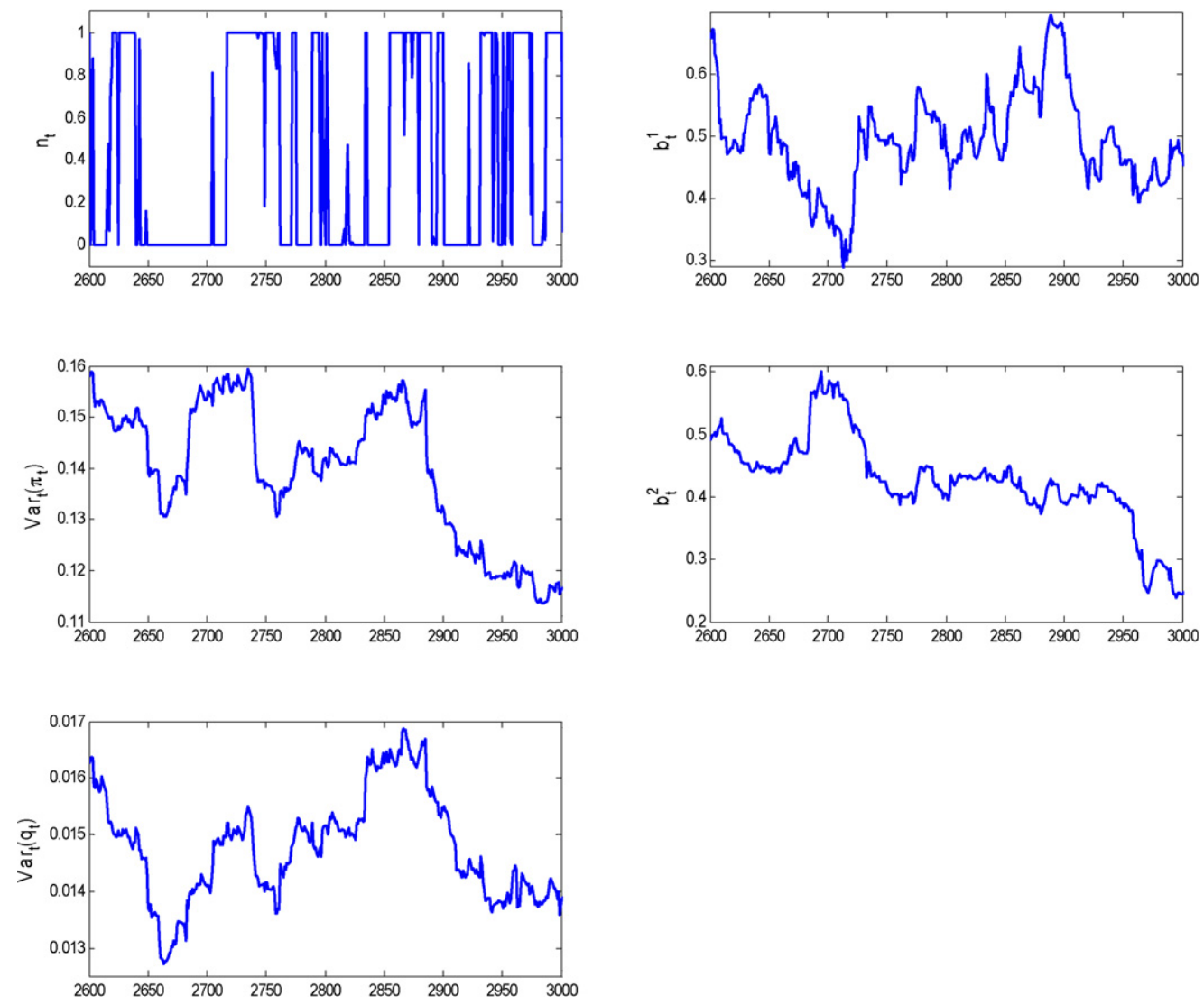

Fig. 9. Parameter learning and dynamic predictor selection with large predictor fitness gain $(\lambda=0.2)$.

reinforces the effect of exogenous disturbances. As agents switch forecasting models, the underlying equilibrium stochastic process changes. This theoretical finding is the basis for the learning and predictor selection dynamics in this section.

The model in this paper is an extension of the learning literature and Brock-Hommes' Adaptively Rational Equilibrium Dynamics (A.R.E.D.). In the current paper beliefs and the choice of forecasting model are jointly determined. In contrast to our earlier paper set in a cobweb modelwhose primary distinction is a negative feedback from beliefs onto the state-we find multiple equilibria. This insight suggested, and our results confirm, that a dynamic version of the model can lead to new and important results.

Previous work by Orphanides and Williams (2005a) and Sargent (1999) highlight the role 'perpetual learning' might play in the Great Moderation. But, as has been argued elsewhere, the actual US experience appears to have been regime shifting and drifting volatility. The results of this section suggest a new avenue for exploring how an economy might endogenously generate shifting inflation and output volatility. This section, however, is not a serious calibration or empirical exercise, but rather a conceptual experiment designed to highlight that the model in Section 3 might generate stochastic properties in line with observed time-series. A rigorous check of this hypothesis is beyond the scope of the present paper. 

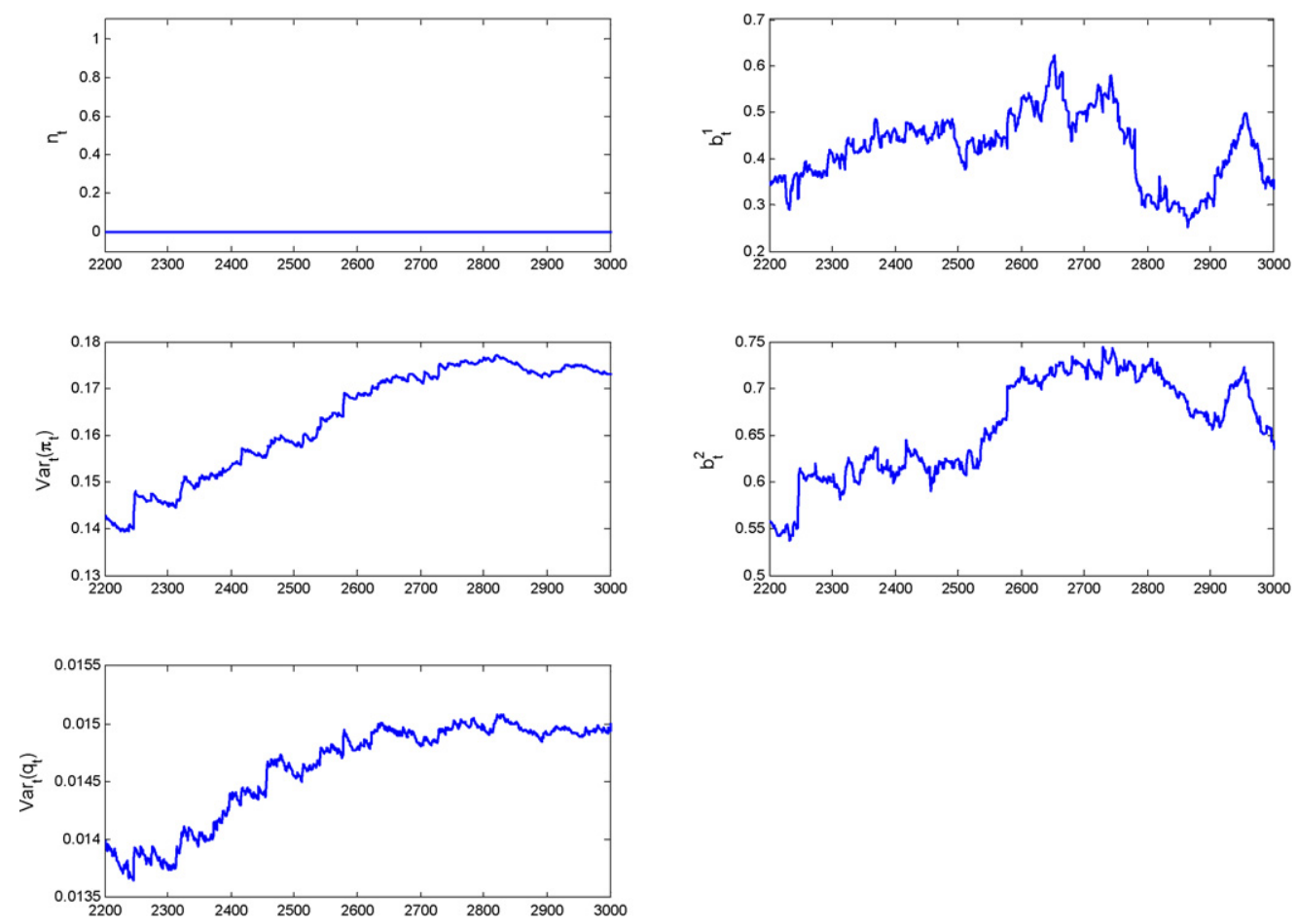

Fig. 10. Parameter learning and dynamic predictor selection with small predictor fitness gain $(\lambda=0.01)$.

In particular, we identify two channels. Parameter learning with a constant gain version of least squares produces drifting volatility, but does not generate regime shifting volatility (Fig. 6). However, the inclusion of constant gain dynamic predictor selection, in which agents estimate a geometric average of past squared forecast errors for each competing model, can lead to distinct shifts in inflation and output volatility (Figs. 7-9). As with constant gain parameter updating, the use of constant gain in estimates of predictor fitness can be interpreted as a way of providing robustness against structural change.

With dual constant gain learning, shocks can occasionally lead agents to switch forecast models. This, via the feedback of expectations onto the state, produces a regime switch in inflation and output volatility that can have varying durations. Evidence presented in Cogley and Sargent (2005a) and Sims and Zha (2006) suggest that drifting and regime switching volatility are important elements of the empirical record. The simulation results in this section demonstrate that a simple self-referential economic model, in which agents choose between competing parsimonious predictors, provides a possible explanation for these findings. Future research should explore to what extent the implications of our theoretical model can account empirically for these characteristics of US time series. Importantly, the gain on past forecast errors plays a central role in the degree to which volatility displays drifting as opposed to regime switching volatility.

It is important to emphasize how natural the assumptions are that generate these results. We model agents as econometricians, in effect, taking the motivation of the learning literature seriously. Because of computational limitations and degrees of freedom problems agents are forced to underparameterize by omitting at least one variable and/or lag from their forecasting model. Although the agents are boundedly rational, they are 'in the spirit' of Muth's original hypothesis 
since agents only select best-performing statistical models. In the real-time dynamic version of the model we again assume that agents behave as econometricians by recursively updating parameter and goodness of fit estimates in light of new data and remaining vigilant against structural change.

\section{Conclusion}

This paper has considered a simple Lucas-type monetary model in which inflation is driven by an exogenous process and by expectations of current inflation. We introduce model uncertainty and underparameterization to the framework. We assume that agents choose the best performing statistical models from a list of misspecified forecasting functions. When agents' predictor choices are endogenous to the model, there exists an equilibrium for the stochastic process, agents' beliefs, and the proportion of agents using a given model. Moreover, there may exist multiple Misspecification Equilibria, each with distinct stochastic properties. Numerical simulations show that a subset of these equilibria are stable under least squares learning. If agents adopt dual learning with constant gains, then the system can endogenously switch between equilibria producing time-varying inflation and output volatility.

There is empirical evidence of time-varying inflation and GDP volatility that is consistent with the equilibrium and real-time learning properties of our model. Importantly, we identify two channels through which the economy may generate endogenously drifting and regime-switching economic volatility. The first channel is drifting parameter estimates that arise from an adaptive learning rule alert to possible structural change. Drifting parameter estimates imply mean forecasts consistent with their equilibrium values, but with occasional departures that induce economic volatility not present in long-run equilibrium. The second channel is dynamic predictor selection. Analogously, predictor selection rules that remain alert to possible structural change can lead agents to switch forecast rules in response to occasional large shocks. Such shocks can induce switching between equilibria and produce persistent swings in inflation and output volatility.

Our results show that endogenous volatility may arise naturally if underparameterization and positive expectational feedback are important elements of the economic process. Strikingly, we are able to obtain these results in a simple Lucas-type model that has a unique rational expectations equilibrium. More generally, the results of this paper therefore indicate that there are potentially important implications from incorporating dual learning of parameter estimates and dynamic forecasting model selection.

\section{Acknowledgments}

We are indebted to the Editor, Associate Editor and a referee for valuable comments. Support from National Science Foundation Grant SES-0617859 is gratefully acknowledged.

\section{Appendix A}

\section{A.1. Derivation of the reduced-form equations}

This appendix derives the reduced-form equations from a yeoman farmer type model with money in the utility function and a fraction of preset prices as studied in Chapter 3.1 of Woodford 
(2003), but extended to include two types of agents who differ by their expectation operator. ${ }^{18}$ Each farmer $i$ uses its own differentiated labor input $N_{i t}$ to produce a good according to the production technology $y_{i t}=\Omega_{t}^{-1 /(1+\eta)} N_{i t}$, where $\Omega_{t}^{1 /(1+\eta)}$ is the unit labor requirement. The farmer then chooses its optimal sequence of consumption, real money and bond holdings (denoted $B_{t}$ ). That is, households solve

$$
\max _{\left\{c_{t}^{i}, m_{t}^{i}, N_{t}^{i}, B_{t}^{i}\right\}} \hat{E}_{0}^{i} \sum_{t=0}^{\infty} \beta^{t} \frac{\left(a_{t} c_{i t}\right)^{1-\gamma}+\left(b_{t} m_{i t}\right)^{1-\gamma}}{1-\gamma}-\frac{\psi_{t}\left(N_{i t}\right)^{1+\eta}}{1+\eta}
$$

subject to $c_{i t}+m_{i t}+B_{i t}=y_{i t}+\frac{p_{t-1}}{p_{t}} m_{i t-1}+\frac{p_{t-1}}{p_{t}}\left(1+i_{t-1}\right) B_{i t-1}$ where $a_{t}, b_{t}, \psi_{t}$ are preference shocks, $i_{t}$ is the nominal one-period interest rate on debt, and $c_{i}, p$ are CES indexes. In the main text $p_{t}, m_{t}$ refer to the logs of the price level and money supply. In this appendix we will instead use $p_{t}$ and $m_{t}$ for the price-level and real money supply, respectively, and use $\hat{p}_{t}$ and $\hat{m}_{t}-\hat{p}_{t}$ to refer to these variables in log form.

The household's first-order conditions can be written as,

$$
\begin{aligned}
& \left(a_{t} c_{i t}\right)^{-\gamma}=\psi \Omega_{t} y_{i t}^{\eta}, \\
& \left(a_{t} c_{i t}\right)^{-\gamma}=\left(b_{t} m_{i t}\right)^{-\gamma}+\beta \hat{E}_{t}^{i}\left(a_{t+1} c_{i t+1}\right)^{-\gamma} \frac{p_{t}}{p_{t+1}}, \\
& \left(a_{t} c_{i t}\right)^{-\gamma}=\beta\left(1+i_{t}\right) \hat{E}_{t}^{i}\left(a_{t+1} c_{i t+1}\right)^{-\gamma} \frac{p_{t}}{p_{t+1}} .
\end{aligned}
$$

These conditions must be satisfied for all $i$ and in all $t$. In the steady-state $p_{t+1} / p_{t}=1$ and $\beta\left(1+i_{t}\right)=1$. Combining the Euler equations it is possible to solve for the money-demand function:

$$
m_{i t}=\left(\frac{i_{t}}{1+i_{t}}\right)^{-1 / \gamma} c_{i t} \frac{a_{t}}{b_{t}}
$$

Following Walsh, and letting $\gamma \rightarrow \infty$, we get

$$
m_{i t}=c_{i t} \frac{a_{t}}{b_{t}} \text {. }
$$

Equilibrium in the money-market requires

$$
m_{t}=n c_{1 t} \frac{a_{t}}{b_{t}}+(1-n) c_{2 t} \frac{a_{t}}{b_{t}}=y_{t} \frac{a_{t}}{b_{t}} .
$$

Taking $\log$ s of both sides

$$
\hat{m}_{t}-\hat{p}_{t}-\hat{a}_{t}+\hat{b}_{t}=\hat{y}_{t}
$$

where $\hat{x}$ denotes the $\log$ of $x$. This is the AD equation.

Firms set price to maximize profits. ${ }^{19}$ Let $\mathcal{P}_{t}$ be the firm's price taking as given the aggregate price-index $p_{t}$. Then a firm's profit function is

$$
\Pi=\mathcal{P}_{t} y_{i t}-\frac{\psi_{t} \Omega_{t} y_{i t}^{1+\eta} p_{t}}{\left(a_{t} c_{i t}\right)^{-\gamma}}
$$

\footnotetext{
18 A more detailed appendix is available in the working paper version.

19 Equivalently, one could replace $y_{i t}$ in the households problem with market demand and let the farmer choose its price.
} 
It can be verified that the F.O.C. reduces to,

$$
\left(\frac{\mathcal{P}_{t}}{p_{t}}\right)^{1+\theta \eta}=\frac{\theta}{\theta-1} \frac{\Omega_{t} \psi_{t} y_{t}^{\eta}}{\left(a_{t} c_{i t}\right)^{-\gamma}},
$$

or, in $\log$ form

$$
\ln \left(\mathcal{P}_{t}\right)=\ln \left(p_{t}\right)+\xi_{y} \ln \left(y_{t}\right)+\xi_{c} \ln \left(c_{i t}\right)+\zeta_{t} .
$$

The variable $\zeta_{t}$ collects the stochastic terms $\Omega_{t}, \psi_{t}, a_{t}$. Following Woodford, assume there is a fraction $\tau$ of firms that set prices optimally in every period, while the remaining set their prices one period in advance. Denote $p_{i f}, p_{i d}$ as the prices of an agent of type $i$ with flexible and predetermined prices, respectively. Then the log-linearized pricing equations are:

$$
\begin{aligned}
& \ln p_{i f t}=\ln p_{t}+\xi_{y} \hat{y}_{t}+\xi_{c} \hat{c}_{i t}+\zeta_{t}, \\
& \ln p_{i d t}=\hat{E}_{t-1}^{i} \ln p_{i f t} .
\end{aligned}
$$

Let $n$ be the fraction of type 1 agents. Defining $\hat{E}_{t-1} \ln p_{t}=n \hat{E}_{t-1}^{1} \ln p_{t}+(1-n) \hat{E}_{t-1}^{2} \ln p_{t}$ we have

$$
\ln p_{t}-\hat{E}_{t-1} \ln p_{t}=\frac{\tau}{1-\tau}\left(\xi_{y}+\xi_{c}\right)\left(\hat{y}_{t}+\zeta_{t}\right) .
$$

Therefore, we have the AS (aggregate supply) relation

$$
\hat{y}_{t}=\phi\left(\hat{p}_{t}-\hat{p}_{t}^{e}\right)+\zeta_{t} .
$$

Appropriately defining $w_{t}$ and $z_{t}=A z_{t-1}+\varepsilon_{t}$, where $z^{\prime}=\left(z_{1}, z_{2}\right)$, in terms of the preference shocks, both the AD and AS equations can be re-written in the same form as the text. In the AS equation we write

$$
q_{t} \equiv \hat{y}_{t}-\hat{\Omega}_{t}=\phi\left(\hat{p}_{t}-\hat{p}_{t}^{e}\right)+\beta_{1} z_{t}
$$

where, for example, $\hat{\Omega}_{t}$ follows a deterministic trend.

\section{A.2. Proofs of propositions}

Proof of Proposition 3. The proof of the proposition follows Lemma 5 in Branch and Evans (2006a). Here we briefly summarize the argument and amend it as necessary. We can rewrite (7) as

$$
S\left(n_{1}\right) \xi=A^{\prime} \gamma,
$$

where $\xi^{\prime}=\left(\xi_{1}, \xi_{2}\right)$ and $S\left(n_{1}\right)$ is the indicated $2 \times 2$ matrix. We seek to sign $\mathrm{d} F / \mathrm{d} n_{1}=$ $(\mathrm{d} F / \mathrm{d} \xi)^{\prime}\left(\mathrm{d} \xi / \mathrm{d} n_{1}\right)$. Following Branch and Evans (2006a), it can be verified that

$$
\begin{aligned}
& \mathrm{d} F / \mathrm{d} n_{1}=2 \theta \xi^{\prime} K\left(n_{1}\right) \xi, \quad \text { where } \\
& K=\left(\begin{array}{cc}
1-\rho \tilde{\rho} & 0 \\
0 & \rho^{2}-Q
\end{array}\right) S^{-1}\left(\begin{array}{cc}
1 & \rho \\
-\tilde{\rho} & -1
\end{array}\right) \\
& =\left(\begin{array}{cc}
\frac{\left(r^{2}-1\right)\left(-1+\left(1+n\left(r^{2}-1\right)\right) \theta\right)}{(1-\theta)+(n-1) n\left(r^{2}-1\right) \theta^{2}} & \frac{\sqrt{Q} r\left(r^{2}-1\right)(\theta-1)}{(1-\theta)+(n-1) n\left(r^{2}-1\right) \theta^{2}} \\
\frac{\sqrt{Q} r\left(r^{2}-1\right)(\theta-1)}{(1-\theta)+(n-1) n\left(r^{2}-1\right) \theta^{2}} & \frac{-Q\left(r^{2}-1\right)\left(1-r^{2} \theta+n\left(r^{2}-1\right) \theta\right)}{(1-\theta)+(n-1) n\left(r^{2}-1\right) \theta^{2}}
\end{array}\right) .
\end{aligned}
$$

Here $r^{2}=\rho \tilde{\rho}$ with $0 \leqslant r^{2}<1$. Notice that $K$ is symmetric. It is easily verified that the necessary and sufficient condition for monotonicity that $K$ is positive semidefinite is satisfied. 
Proof of Proposition 5. Our proof again follows Branch and Evans (2006a). In our earlier paper it was established that for each $\alpha$ the map $T_{\alpha}$ has a fixed point denoted $n^{*}(\alpha)$, and, moreover, $\exists\{\alpha(s)\}_{s}$ s.t. $\alpha(s) \rightarrow \infty \Rightarrow n(\alpha(s)) \rightarrow \bar{n}$ for some $\bar{n}$ which is a fixed point to the map $\lim _{\alpha(s) \rightarrow \infty} T_{\alpha(s)}$. The proposition claims that $\bar{n} \in\{0, \hat{n}, 1\}$ where $F(\hat{n})=0$. That $\hat{n}$ is a fixed point was proven in Proposition 8 of Branch and Evans (2006a). Following the arguments for Conditions P0 and P1 in that proposition, it is clear that $F^{\prime}>0$ implies $\bar{n} \in\{0,1\}$ is a fixed point.

\section{References}

Adam, K., 2005a. Learning to forecast and cyclical behavior of output and inflation. Macroeconomic Dynamics 1, 1-27. Adam, K., 2005b. Experimental evidence on the persistence of output and inflation. Economic Journal. In press.

Bacchetta, P., Van Wincoop, E., 2004. A scapegoat model of exchange rate fluctuations. American Economic Review 94, 114-118.

Bernanke, B.S., Mihov, I., 1998. Measuring monetary policy. Quarterly Journal of Economics 113 (3), 869-902.

Branch, W.A., Evans, G.W., 2006a. Intrinsic heterogeneity in expectation formation. Journal of Economic Theory 127, 264-295.

Branch, W.A., Evans, G.W., 2006b. A simple recursive forecasting model. Economics Letters 91, 158-166.

Branch, W.A., McGough, B., 2005. Misspecification and consistent expectations in stochastic non-linear economies. Journal of Economic Dynamics and Control 29, 659-676.

Branch, W.A., Carlson, J., Evans, G.W., McGough, B., 2006. Monetary policy, endogenous inattention, and the volatility trade-off. Mimeo.

Brock, W.A., Hommes, C.H., 1997. A rational route to randomness. Econometrica 65, 1059-1160.

Brock, W.A., Hommes, C.H., 1998. Heterogeneous beliefs and routes to chaos in a simple asset pricing model. Journal of Economic Dynamics and Control 22, 1235-1274.

Brock, W.A., Durlauf, S.N., West, K.D., 2003. Policy analysis in uncertain economic environments. Brookings Papers on Economic Activity 1, 235-322.

Bullard, J., Cho, I.-K., 2005. Escapist policy rules. Journal of Economic Dynamics and Control 29, 1841-1865.

Chari, V.V., Kehoe, P.J., McGrattan, E.R., 2005. A critique of structural vars using real business cycle theory. W.P. 631. FRB-Minneapolis.

Cho, I.-K., Kasa, K., 2003. Learning dynamics and endogenous currency crises. Mimeo.

Cho, I.-K., Kasa, K. 2006. Learning and model validation. Mimeo.

Cho, I.-K., Williams, N., Sargent, T.J., 2002. Escaping Nash inflation. Review of Economic Studies 69, 1-40.

Cogley, T.W., Sargent, T.J., 2005a. Drifts and volatilities: Monetary policies and outcomes in the post WWII US. Review of Economic Dynamics 8, 262-302.

Cogley, T.W., Sargent, T.J., 2005b. The conquest of US inflation: Learning and robustness to model uncertainty. Review of Economic Dynamics 8, 528-563.

Evans, G.W., Honkapohja, S., 1993. Adaptive expectations, hysteresis and endogenous fluctuations. Federal Reserve Bank of San Francisco Economic Review 1, 3-13.

Evans, G.W., Honkapohja, S., 2001. Learning and Expectations in Macroeconomics. Princeton Univ. Press, Princeton, NJ.

Evans, G.W., Ramey, G., 1992. Expectation calculation and macroeconomic dynamics. American Economic Review 82 (1), 207-224.

Evans, G.W., Ramey, G., 2006. Adaptive expectations, underparameterization and the Lucas critique. Journal of Monetary Economics 53, 249-264.

Evans, G.W., Honkapohja, S., Sargent, T.J., 1989. On the preservation of deterministic cycles when some agents perceive them to be random fluctuations. Journal of Economic Dynamics and Control 17, 705-721.

Fernandez-Villaverde, J., Rubio-Ramirez, J.F., 2006. Estimating macro models: A likelihood approach. Mimeo.

Guse, E., 2005. Stability properties for learning with heterogeneous expectations and multiple equilibria. Journal of Economic Dynamics and Control 29, 1623-1642.

Hansen, L.P., Sargent, T.J., 2005. Misspecification in recursive macroeconomic theory. Manuscript.

Hommes, C., Sorger, G., 1998. Consistent expectations equilibria. Macroeconomic Dynamics 2, 287-321.

Hommes, C., Sorger, G., Wagener, F., 2002. Learning to believe in linearity in an unknown nonlinear stochastic economy. Mimeo. 
Kasa, K., 2004. Learning, large deviations, and recurrent currency crises. International Economic Review 45, $141-173$.

Kim, C.-J., Nelson, C., 1999. Has the US economy become more stable? A Bayesian approach based on a Markovswitching model of the business cycle. Review of Economics and Statistics 81 (4), 608-616.

Kim, C.-J., Nelson, C., Piger, J., 2004. The less volatile US economy: A Bayesian investigation of timing, breadth, and potential explanations. Journal of Business and Economic Statistics 22 (1), 80-93.

Kydland, F.E., Prescott, E.C., 1977. Rules rather than discretion: The inconsistency of optimal plans. Journal of Political Economy 85, 473-491.

Lucas Jr., R.E., 1973. Some international evidence on output-inflation trade-offs. American Economic Review 63, 326334.

Justiniano, A., Primiceri, G., 2006. The time-varying volatility of macroeconomic fluctuations. Mimeo.

Manski, C.F., McFadden, D., 1981. Structural Analysis of Discrete Data with Econometric Applications. MIT Press, Cambridge, MA.

Marcet, A., Nicolini, J.P., 2003. Recurrent hyperinflation and learning. American Economic Review 93 (5), 1476-1495.

Marcet, A., Sargent, T.J., 1989. Convergence of least-squares learning mechanisms in self-referential linear stochastic models. Journal of Economic Theory 48, 337-368.

Marcet, A., Sargent, T.J., 1995. Speed of convergence of recursive least squares: Learning with autoregressive movingaverage perceptions. In: Kirman, A., Salmon, M. (Eds.), Learning and Rationality in Economics. Basil Blackwell, Oxford, pp. 179-215.

McConnell, M., Quiros, G.P., 2000. Output fluctuations in the United States: What has changed since the early 1980s? American Economic Review 90 (5), 1464-1476.

McGough, B., 2006. Shocking escapes. Economic Journal 116, 507-528.

Milani, F., 2005. Expectations, learning, and macroeconomic persistence. Mimeo.

Muth, J.F., 1961. Rational expectations and the theory of price movements. Econometrica 29, 315-335.

Orphanides, A., Williams, J.C., 2005a. Imperfect knowledge, inflation expectations, and monetary policy. Chapter 5 in: Bernanke, B.S., Woodford, M. (Eds.), Inflation Targeting. National Bureau of Economic Research and Univ. of Chicago Press, Chicago.

Orphanides, A., Williams, J.C., 2005b. The decline of activist stabilization policy: Natural rate misperceptions, learning and expectations. Journal of Economic Dynamics and Control 29, 1927-1950.

Owyang, M.T., 2001. Persistence, excess volatility, and volatility clusters in inflation. Federal Reserve Bank of St. Louis Review (Nov./Dec.), 41-52.

Sargent, T.J., 1987. Macroeconomic Theory, second ed. Academic Press, New York.

Sargent, T.J., 1991. Bounded Rationality in Macroeconomics. Oxford Univ. Press, Oxford.

Sargent, T.J., 1999. The Conquest of American Inflation. Princeton Univ. Press, Princeton, NJ.

Sargent, T.J., Williams, N., 2005. Impact of priors on convergence and escape from Nash inflation. Review of Economic Dynamics 8 (2), 360-391.

Sensier, M., van Dijk, D., 2004. Testing for changes in volatility of US macroeconomic time series. Review of Economics and Statistics 86, 833-839.

Sims, C.A., Zha, T., 2006. Were there regime switches in US monetary policy? American Economic Review 96 (1), 54-81.

Stock, J., Watson, M.W., 2003. Has the business cycle changed? Evidence and explanations. In: FRB Kansas City symposium. Jackson Hole, WY, August 28-30, 2003, pp. 9-56.

Walsh, C., 2003. Monetary Theory and Policy, second ed. MIT Press, Cambridge, MA.

Williams, N., 2004a. Escape dynamics in learning models. Mimeo.

Williams, N., 2004b. Stability and long-run equilibrium in stochastic fictitious play. Mimeo.

Woodford, M., 2003. Interest and Prices. Princeton Univ. Press, Princeton, NJ. 